\title{
Adaptive Gain Scheduled Semiactive Vibration Control Using a Neural Network
}

\author{
Kazuhiko Hiramoto $\mathbb{D}^{1},{ }^{1}$ Taichi Matsuoka, ${ }^{2}$ and Katsuaki Sunakoda ${ }^{3}$ \\ ${ }^{1}$ Mechanical Engineering Program, Niigata University, Niigata 950-2181, Japan \\ ${ }^{2}$ Department of Mechanical Engineering Informatics, Meiji University, Kawasaki 214-8571, Japan \\ ${ }^{3}$ Akita University, Akita 010-8502, Japan \\ Correspondence should be addressed to Kazuhiko Hiramoto; hiramoto@eng.niigata-u.ac.jp
}

Received 21 August 2017; Revised 25 October 2017; Accepted 4 December 2017; Published 15 January 2018

Academic Editor: Oren Lavan

Copyright (C) 2018 Kazuhiko Hiramoto et al. This is an open access article distributed under the Creative Commons Attribution License, which permits unrestricted use, distribution, and reproduction in any medium, provided the original work is properly cited.

\begin{abstract}
We propose an adaptive gain scheduled semiactive control method using an artificial neural network for structural systems subject to earthquake disturbance. In order to design a semiactive control system with high control performance against earthquakes with different time and/or frequency properties, multiple semiactive control laws with high performance for each of multiple earthquake disturbances are scheduled with an adaptive manner. Each semiactive control law to be scheduled is designed based on the output emulation approach that has been proposed by the authors. As the adaptive gain scheduling mechanism, we introduce an artificial neural network (ANN). Input signals of the ANN are the measured earthquake disturbance itself, for example, the acceleration, velocity, and displacement. The output of the ANN is the parameter for the scheduling of multiple semiactive control laws each of which has been optimized for a single disturbance. Parameters such as weight and bias in the ANN are optimized by the genetic algorithm (GA). The proposed design method is applied to semiactive control design of a base-isolated building with a semiactive damper. With simulation study, the proposed adaptive gain scheduling method realizes control performance exceeding single semiactive control optimizing the average of the control performance subject to various earthquake disturbances.
\end{abstract}

\section{Introduction}

Semiactive vibration control of structural systems with ER, MR dampers, or variable stiffness device, and so on can realize control performance exceeding the passive control, that is, structural modification including the addition of tuned mass dampers to the main structural system. Moreover, semiactive control is simple, has low energy consumption, and is safe against failure of the control element compared to active control that is realized by sensors, a (feedback) controller, and actuators requiring a large power supply. Because of the above advantages, semiactive control has been actively studied as a vibration control method for structural systems subject to large-scale earthquake disturbances in which surrounding power failure can be occurred [1-5].

One of the most important specifications that the semiactive control system should satisfy is that the designed semiactive control law has high control performance against future unknown seismic disturbances. In order to satisfy the specification, design parameters in the semiactive control law are optimized to achieve good control performance against recorded and/or artificial earthquake disturbances having various time and frequency characteristics. In designing a single time-invariant control law that does not depend on the disturbance characteristics, the semiactive control system is necessary to realize the averagely good control performance on vibration suppression for earthquake disturbances with various time and frequency characteristics. It means that the semiactive control law must satisfy the performance robustness with respect to the variation of the earthquake disturbance and the realizable control performance of the single semiactive control law is limited because of the performance robustness itself.

To overcome such a situation, if we can adaptively change the semiactive control law depending on the characteristics of the earthquake disturbance, higher control performance 
than that of the single control law described above, that is, the single control law optimizing the control performance on average for all disturbances, is expected to be realized. As an example of the adaptive change, the following method can be considered:

(1) We firstly design multiple control laws for seismic disturbances with some different properties. Each of the semiactive control laws is designed to maximize control performance only for a single disturbance with a specific property.

(2) For the future unknown earthquake disturbance, the best semiactive control law is selected from or interpolated with the multiple semiactive control laws designed in the previous step. The selection or interpolation is determined based on available sensor data, for example, the earthquake disturbance.

Regarding the characteristic analysis of earthquake disturbance, many methodologies in the time and/or frequency domain have been proposed from the viewpoint of seismology. However, we cannot determine what kind of characteristics of the earthquake wave should be considered as a reference for selecting the most appropriate semiactive control law from multiple candidates of the control law (or interpolating multiple semiactive control laws) to obtain the good control performance.

In this study, we adopt an artificial neural network (ANN) as an approach to make appropriate selection or interpolation of the above-mentioned multiple semiactive control laws. Artificial neural networks have been widely used for pattern recognition, modeling, and control system design during some decades [6]. Recently, along with the development of techniques such as deep learning, the application of the neural network to artificial intelligence has become actively studied. In this paper, we design an ANN with an input of a measured earthquake disturbance and an output of parameters to schedule multiple semiactive control laws with different properties. Parameters in the ANN, for example, weighting and bias, are optimized so that the ANN can be used as a good adaptive gain scheduler to interpolate multiple semiactive control laws from earthquake disturbance information. We demonstrate the effectiveness of the proposed method by simulation using the adaptive gain scheduled semiactive control law with the ANN-based scheduling mechanism.

The rest of the paper is organized as follows. In Section 2, a structural model with semiactive control devices is defined. In Section 3, the detail of the methodology of the gain scheduled semiactive control system composed of the multiple semiactive control laws and the ANN-based scheduler are described. The simulation study is given in Section 4 and the conclusion of the study is shown in Section 5.

\section{Structural Model}

The control object in the study is a $n$-DOF structural system that has $n_{s}$ semiactive control devices whose damping coefficients can be changed with command signals shown in

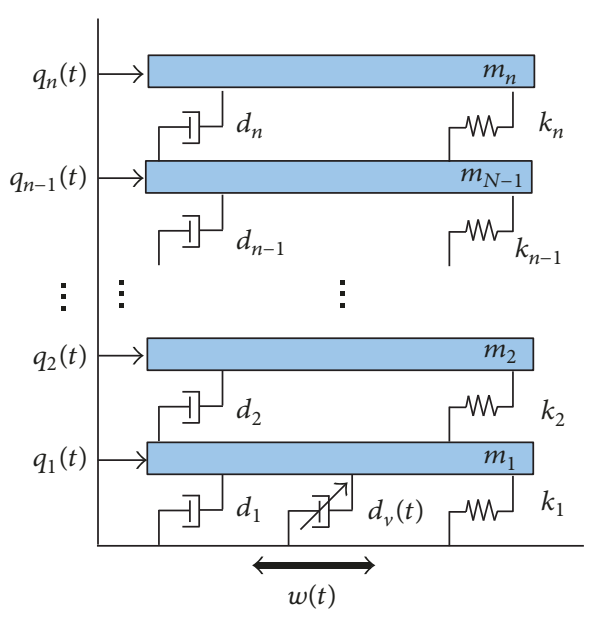

FIGURE 1: $n$-DOF structural system with $n_{s}$ semiactive dampers.

Figure 1. The equation of motion and the state-space form of the control object are given as follows:

$$
\begin{gathered}
M \ddot{q}(t)+\left(D_{0}+\sum_{j=1}^{n_{s}} d_{j}^{s}(t) D_{j}^{s}\right) \dot{q}(t)+K q(t) \\
=G w(t)+\left(H_{0}+\sum_{j=1}^{n_{s}} d_{j}^{s}(t) H_{j}^{s}\right) \dot{w}(t) \\
q(t)=\left[\begin{array}{llll}
q_{1}(t) & q_{2}(t) & \cdots & q_{n}(t)
\end{array}\right]^{T},
\end{gathered}
$$

where $q_{i}(t), i=1, \ldots, n$, and $w(t)$ are the absolute displacement of $i$-th mass (floor) and the earthquake disturbance, respectively. Matrices $M=M^{T}>0, D_{0}=D_{0}^{T}>0$, and $K=K^{T}>0$ are the structural mass, damping, and stiffness matrices, respectively. Matrices $G$ and $H_{0}$ are the influence coefficient matrices.

The state-space model of the structural system defined in (1) is given by

$$
\begin{aligned}
& \dot{x}(t)=\left(A_{0}+\sum_{j=1}^{n_{s}} d_{j}^{s}(t) A_{j}^{s}\right) x(t) \\
& +\left(B_{0}+\sum_{j=1}^{n_{s}} d_{j}^{s}(t) B_{j}^{s}\right) v(t) \\
& z(t)=C_{z} x(t)+D_{z} v(t) \\
& y(t)=C_{y} x(t)+D_{y} v(t), \\
& x(t)=\left[\begin{array}{ll}
q^{T}(t) & \dot{q}^{T}(t)
\end{array}\right]^{T}, \\
& v(t)=\left[\begin{array}{ll}
w(t) & \dot{w}(t)
\end{array}\right]^{T}, \\
& A_{0}=\left[\begin{array}{cc}
0_{n \times n} & I_{n} \\
-M^{-1} K & -M^{-1} D_{0}
\end{array}\right] \text {, }
\end{aligned}
$$




$$
\begin{aligned}
& A_{j}^{s}=\left[\begin{array}{c}
0_{n \times 2 n} \\
0_{n \times n}-M^{-1} D_{s}^{j}
\end{array}\right], \quad j=1, \ldots, n_{s},
\end{aligned}
$$

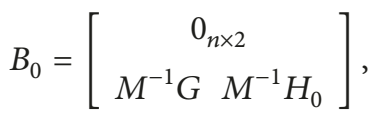

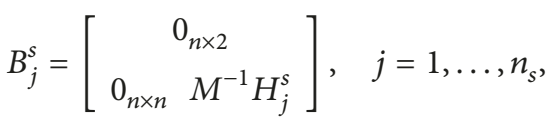

where $z(t)$ and $y(t)$ are the controlled output for the performance evaluation and the measurement vector obtained with sensors installed on the structural system.

The variable damping coefficient of the $j$ th semiactive damper $d_{j}^{s}(t)$ is varied in a range given as follows:

$$
0 \leq \underline{d_{j}^{s}} \leq d_{j}^{s}(t) \leq \overline{d_{j}^{s}}, \quad j=1, \ldots, n_{s},
$$

where $\overline{d_{j}^{s}}$ and $d_{j}^{s}$ are the maximum and minimum values of the variable damping coefficient of the $j$ th semiactive damper, respectively.

Moreover, there exists a dynamic delay in the damping coefficient of the semiactive damper for its command signal. The characteristics of the dynamic delay are modeled as follows:

$$
\begin{aligned}
& \dot{x}_{j}^{s}(t)=-\frac{1}{T_{j}^{s}} x_{j}^{s}(t)+\frac{1}{T_{j}^{s}} d_{j}^{c}(t) \\
& d_{j}^{s}(t)=s_{j}\left(x_{j}^{s}(t)\right),
\end{aligned}
$$

$$
j=1, \ldots, n_{s},
$$

where $T_{j}^{s}$ and $d_{j}^{c}(t), j=1, \ldots, n_{s}$, are the time constant and the command signal of the $j$ th semiactive damper, respectively. The function $s_{j}(\cdot), j=1, \ldots, n_{s}$, is a saturation function defined as

$$
s_{j}\left(x_{j}^{s}\right)= \begin{cases}\overline{d_{j}^{s}} & \left(x_{j}^{s} \geq d_{j}^{s}\right) \\ x_{j}^{s} & \left(\underline{d_{j}^{s}}<x_{j}^{s}<\overline{d_{j}^{s}}\right) \quad j=1, \ldots, n_{s} . \\ \underline{d_{j}^{s}} & \left(x_{j}^{s} \leq \underline{d_{j}^{s}}\right),\end{cases}
$$

\section{Control System Design}

3.1. Adaptive Gain Scheduling. It is well-known that seismic waves have common characteristics to some extent in time and frequency domain $[7,8]$. On the other hand, there are many different characteristics between seismic waves. The difference is due to many factors such as the mechanism of the earthquake, the distance between the seismic center and the controlled structural system, and the propagation path of the seismic wave.

Generally, structural vibration control systems subject to earthquakes are designed in consideration of the commonality of the above frequency characteristics. In other words, if the vibration control law is designed so as to exhibit good control performance against typical disturbance, then, from the commonality point of view, the control system is expected to achieve good control performance for unknown earthquake disturbances including future seismic events.

However, it is also a fact that the achievable performance of the vibration control system changes depending on characteristics of the earthquake disturbance. For example, when a vibration control system that optimizes the design parameters so as to exert good control performance for the disturbance $A$ receives a disturbance $B$ that is different in time and frequency characteristics from the disturbance $A$, the performance of the control system is not necessarily equivalent to that exerted against disturbance $A$.

In order to deal with the performance variation of the control system, the design parameters of the control law for determining the control characteristics are optimized for multiple recorded and/or artificial earthquakes (that are used as test disturbance signals for the simulation of the control system) so that the control performance becomes good on average in the general design process of the vibration control system. This concept can be referred to as a robust performance concept for various characteristics of earthquake disturbance. However, taking a robust performance approach, control performance against the individual earthquake tends to be limited due to the robust performance property of the control law itself.

Remark 1. In control community, the concept of the robust performance is dealt with more mathematically with the notion of the structured singular value of the closed-loop system [9]. However, the authors dare to use the term "robust performance" here because the control design for multiple disturbance aims at the robust performance of the control system from the practical viewpoint.

Contrary to the robust performance approach described above, a method that achieves optimum control performance by adaptively scheduling multiple control laws can be considered. Each of the control laws in the approach has been obtained in advance so as to have high control performance against a specific earthquake disturbance. Because this control concept does not require consideration of the robust performance specifications, further improvement in control performance can be expected.

In this study, we propose a mechanism to adaptively schedule multiple semiactive control laws with different control characteristics based on information such as earthquake disturbance obtained from sensors. The block diagram of the control system is shown in Figure 2, where $P, C_{k}$, $k=1, \ldots, n_{c}$, are the structural system to be controlled and a semiactive control law with high control performance against a specific earthquake disturbance, respectively. The input to $C_{k}, k=1, \ldots, n_{c}$, is the structural response $y(t)$ obtained from the sensor, and the output is the command signal of the semiactive damper denoted by a vector $u_{k}^{c}=$ $\left[\left(d_{1}^{c}\right)_{k} \cdots\left(d_{n_{s}}^{c}\right)_{k}\right]^{T}, k=1, \ldots, n_{c}$, where $j$-th element of the vector $u_{k}^{c}$ is the command signal to change the variable 


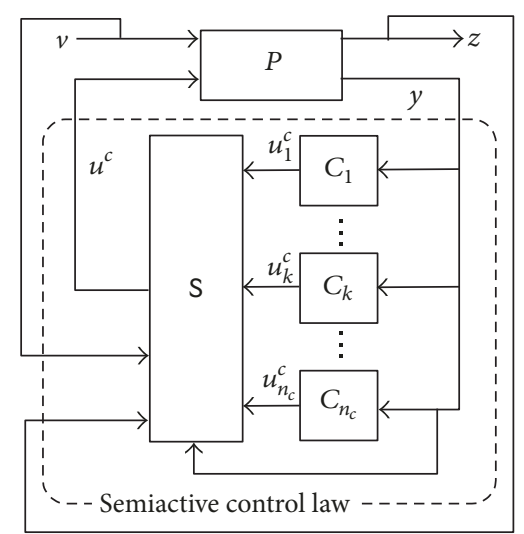

FIGURE 2: Block diagram of the adaptive gain scheduling semiactive control system with $C_{k}, k=1, \ldots, n_{c}$, and the scheduling mechanism S.

damping coefficient $d_{j}^{s}(t), j=1, \ldots, n_{s}$ (defined in (6)), by the $k$ th semiactive control law $C_{k}$. The block $S$ is an adaptive gain scheduling mechanism that determines the most appropriate selection or interpolation of all $u_{k}^{c}(t)$ 's generated by $n_{c}$ semiactive control laws $C_{k}, k=1, \ldots, n_{c}$, at the time instant $t$. The scheduled command signal of $n_{s}$ semiactive dampers is defined as $u^{c}=\left[\begin{array}{lll}d_{1}^{c} & \cdots & d_{n_{s}}^{c}\end{array}\right]^{T}$. In principle, the adaptive scheduling mechanism $S$ can utilize sensor information used by the semiactive control law and command signal $u^{c}(t)$ itself.

In the next two subsections, we describe the detail of the design method of the feedback control law $C_{k}$ and the adaptive scheduling mechanism, the block $S$ in Figure 2.

3.2. Design of the Semiactive Control Law $C_{k}$. The semiactive control law $C_{k}, k=1, \ldots, n_{c}$, in the previous subsection is designed to achieve high control performance against a specific single earthquake disturbance. The block diagram for designing the semiactive control law $C_{k}, k=1, \ldots, n_{c}$, is shown in Figure 3 , where $v_{k}, k=1, \ldots, n_{c}$, is the specific single earthquake disturbance considered during the design of the semiactive control law $C_{k}$. The adjustable design parameters in the semiactive control law $C_{k}$ are optimized so that the closed-loop system consisting of the control object $P$ and the semiactive control law $C_{k}$ exhibits high control performance under the earthquake disturbance $v_{k}$.

In this study, the semiactive control law $C_{k}$ is designed using the output emulation approach [5] recently proposed by the authors. In the output emulation method, the command signal of the variable damping coefficient of the semiactive damper is switched between the maximum and minimum values. The switching criterion is the error between the predicted controlled outputs of the semiactive control and reference active systems. In the following, the detail of the output emulation approach will be described in detail.

The reference active control system can be obtained by replacing the semiactive dampers of the control object in (3) with force actuators. The reference active control system is a virtual system that cannot be realized in the present situation

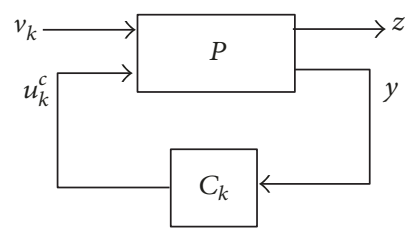

FIGURE 3: Block diagram of the control system for the synthesis of the semiactive control law $C_{k}$.

but is used as the performance target of the semiactive control system. The state-space form of the reference active control system is defined as follows:

$$
\begin{aligned}
\dot{x}_{a}(t) & =\mathrm{A}_{0} x_{a}(t)+B_{0} v(t)+B_{a} u^{a}(t) \\
z_{a}(t) & =C_{z} x_{a}(t)+D_{z} v(t) \\
y(t) & =C_{y} x_{a}(t)+D_{y} v(t), \\
x_{a}(t) & =\left[\begin{array}{ll}
q^{T}(t) & \dot{q}^{T}(t)
\end{array}\right]^{T}, \\
v(t) & =\left[\begin{array}{ll}
w(t) & \dot{w}(t)
\end{array}\right]^{T},
\end{aligned}
$$

where $x_{a}(t)$ and $u^{a}(t)=\left[\begin{array}{lll}u_{1}^{a}(t) & \cdots & u_{n_{s}}^{a}\end{array}\right]^{T}$ are the state vector and the control force vector of the reference active control system, respectively. Note that the controlled output $z_{a}(t)$ of the reference active control system has the same components as those of $z(t)$ of the control object in (3).

In this study, we assume the state vector $x_{a}(t)$ is available with sensors; that is, the measured output in (8) is $y(t)=$ $x_{a}(t)$. Under the assumption, we define the reference active control law as a state-feedback given by

$$
u_{a}(t)=-K_{a} x_{a}(t),
$$

where $K_{a}$ is the state-feedback gain matrix of the reference active control law. The closed-loop system with the reference active control system in (8) and the state-feedback control law in (10) becomes

$$
\begin{aligned}
& \dot{x}_{a}(t)=A_{c} x_{c}(t)+B_{0} v(t) \\
& z_{a}(t)=C_{z} x_{c}(t)+D_{z} v(t),
\end{aligned}
$$

$$
A_{c}=A_{0}-B_{a} K_{a} .
$$

In the output emulation approach, the command signal to change the variable damping coefficient of the semiactive damper is changed with a measure of the closeness of the controlled output between the semiactive and reference active control systems. The closeness between the semiactive and reference active control systems is evaluated by using the predicted controlled output of two systems. The detail of the process to obtain the command signal of the semiactive damper in the output emulation approach is described below.

Let $t_{0}$ and $\Delta t>0$ be the current time and the small prediction length, respectively. From (11), the predicted control output of the reference active control is given as

$$
z_{a}\left(t_{0}+\Delta t\right)=C_{z} x_{a}\left(t_{0}+\Delta t\right)+D_{z} v\left(t_{0}+\Delta t\right) .
$$


The predicted value of the disturbance vector at $t=t_{0}+\Delta t$ can be approximately obtained by

$$
\begin{aligned}
v\left(t_{0}+\Delta t\right) & \simeq v\left(t_{0}\right)+\Delta t \dot{v}\left(t_{0}\right) \\
& =\left[\begin{array}{l}
\dot{w}\left(t_{0}\right) \\
w\left(t_{0}\right)
\end{array}\right]+\Delta t\left[\begin{array}{l}
\ddot{w}\left(t_{0}\right) \\
\dot{w}\left(t_{0}\right)
\end{array}\right] .
\end{aligned}
$$

Similarly the predicted state vector of the reference active control system at $t=t_{0}+\Delta t$ is approximately given as follows:

$$
x_{a}\left(t_{0}+\Delta t\right) \simeq x_{a}\left(t_{0}\right)+\Delta t \dot{x}_{a}\left(t_{0}\right) .
$$

With (11), we have the time derivative of $x_{c}(t)$ at $t=t_{0}$ as

$$
\dot{x}_{a}\left(t_{0}\right)=A_{c} x_{a}\left(t_{0}\right)+B_{0} v\left(t_{0}\right) .
$$

In order to get $\dot{x}_{a}\left(t_{0}\right)$, we can adopt numerically stable integration methods such as the Runge-Kutta method and Newmark's $\beta$ method. The predicted control output vector $z_{a}\left(t_{0}+\Delta t\right)$ of the reference active control system can be obtained by (12)-(15). With the continuing measurement of $x_{a}\left(t_{0}\right)$ and $v\left(t_{0}\right)$, we can obtain the predicted output signal of the reference active control system.

Similarly, we can obtain the predicted control output of the semiactive system. The predicted control output of the semiactive system $z\left(t_{0}+\Delta t\right)$ is given as

$$
z\left(t_{0}+\Delta t\right)=C x\left(t_{0}+\Delta t\right)+D v\left(t_{0}+\Delta t\right) .
$$

The predicted value of the disturbance vector $v\left(t_{0}+\Delta t\right)$ can be obtained with (13). The predicted state vector of the semiactive control system is given as

$$
x\left(t_{0}+\Delta t\right) \simeq x\left(t_{0}\right)+\Delta t \dot{x}\left(t_{0}\right) .
$$

With (3), (5), and (6), the time derivative of the state vector of the $j$ th semiactive control system at $t=t_{0}$ is obtained as follows:

$$
\begin{aligned}
\dot{x}_{j}^{s}\left(t_{0}\right)= & -\frac{1}{T_{j}^{s}} x_{j}^{s}\left(t_{0}\right)+\frac{1}{T_{j}^{s}} d_{j}^{c}\left(t_{0}\right) \\
d_{j}^{s}\left(t_{0}\right)= & s_{j}\left(x_{j}^{s}\left(t_{0}\right)\right), \quad j=1, \ldots, n_{s}, \\
\dot{x}\left(t_{0}\right)= & \left(A_{0}+\sum_{j=1}^{n_{s}} d_{j}^{s}\left(t_{0}\right) A_{j}^{s}\right) x\left(t_{0}\right) \\
& +\left(B_{0}+\sum_{j=1}^{n_{s}} d_{j}^{s}\left(t_{0}\right) B_{j}^{s}\right) v\left(t_{0}\right) .
\end{aligned}
$$

When we fix all the command signals of the semiactive damper at $t=t_{0}$, we can obtain $\dot{x}\left(t_{0}\right)$. As the candidates of the command signal of the semiactive damper at $t=$ $t_{0}$, we assume the maximum and minimum values of the variable damping coefficient of the semiactive damper given as follows:

$$
d_{j}^{c}\left(t_{0}\right)=\left\{\begin{array}{l}
\overline{d_{v}^{j}} \\
\underline{d_{v}^{j}},
\end{array} \quad j=1, \ldots, n_{s} .\right.
$$

For all possible command signal combinations, we can calculate the predicted control output of the semiactive control system at $t=t_{0}+\Delta t$ with (13) and (16)-(18). For the bang-bang type command signal in (19), the number of $n_{s}$ command signals' combinations is $2^{n_{s}}$.

In the output emulation approach, the command signal of the $j$ th semiactive damper at $t=t_{0}$ is selected from $2^{n_{s}}$ combinations of command signals (shown in (19)) in real time as follows:

$$
\begin{aligned}
d_{j}^{c}\left(t_{0}\right)=\underset{d_{j}^{c}\left(t_{0}\right)=\overline{d_{j}^{c}} \text { or } \underline{d_{j}^{c}}}{\operatorname{argmin}} J_{e}\left(t_{0}\right), \\
J_{e}=\left\|z_{a}\left(t_{0}+\Delta t\right)-z\left(t_{0}+\Delta t\right)\right\|, j=1, \ldots, n_{s} .
\end{aligned}
$$

Remark 2. In the present study, the state vector in (3) is composed of the absolute displacement and velocity of each floor. In conventional modeling of the civil structural system subject to earthquake disturbance, the state vector is defined as the relative displacement and velocity between each floor and ground motion given by

$$
x_{c}=\left[\begin{array}{c}
q(t)-\mathbf{1} w(t) \\
\dot{q}(t)-\mathbf{1} \dot{w}(t)
\end{array}\right], \quad \mathbf{1}=\underbrace{[1 \cdots 1]^{T}}_{n} .
$$

In this case, the disturbance is defined as $\ddot{w}(t)$, the acceleration of the earthquake wave. In the output emulation approach, predicted values of disturbance velocity and displacement are obtained by using (13) in order to obtain the predicted value of the controlled output $z\left(t_{0}+\Delta t\right)$. In general, earthquake waves are often measured by an acceleration sensor. The velocity and displacement of the disturbance can be obtained by performing numerical integration of sensor measurement $\ddot{w}(t)$. Then, in the state-space model of the present study, the predicted disturbance signal $v\left(t_{0}+\Delta t\right)$ is obtained. On the other hand, if the output emulation method is applied to the conventional model whose disturbance term is defined as the acceleration, the jerk which is the time derivative of the acceleration is required in order to obtain the predicted value of the disturbance in a similar way to that of (13). The numerical differentiation of the measured disturbance acceleration is sometimes not appropriate because it may be contaminated with the sensor noise in the higherfrequency range. Therefore, the state-space form as (3) is employed in this study.

Moreover, the predicted controlled output signals in (12) and (16) are the 1st-order approximation of the Taylor series expansion of $z_{a}\left(t_{0}+\Delta t\right)$ and $z\left(t_{0}+\Delta t\right)$. In the ideal noisefree situation, higher order time derivative terms of the disturbance signal, for example, $d^{3} w(t) / d t^{3}$ and $d^{4} w(t) / d t^{4}$, work to improve the accuracy of the approximation of $z_{a}\left(t_{0}+\right.$ $\Delta t)$ and $z\left(t_{0}+\Delta t\right)$. However, it is not necessarily true in the real situation because of the sensor noise problem shown in the above paragraph. This is the reason why the 1st-order approximation of the controlled output is used.

In the output emulation approach, the characteristics of closed-loop reference active control system in (11) are adjusted to optimize the control performance of the semiactive control system. In this study, we adopt the state-feedback 


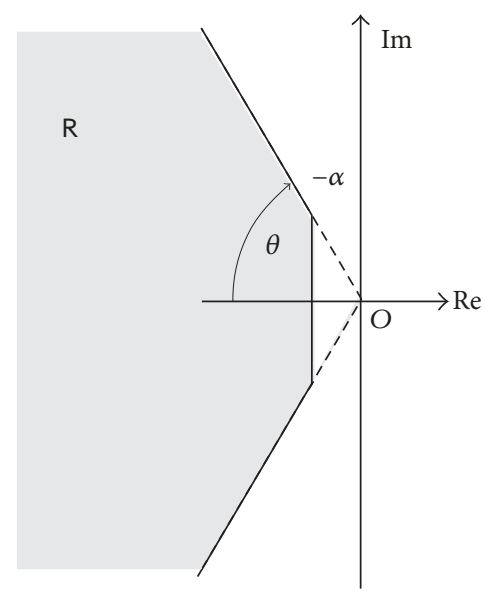

FIGURE 4: Region R in the complex plane.

gain matrix $K_{a}$ of the reference active control law in (10) so that the closed-loop poles locate in a region $\mathrm{R}$ in the complex plane shown in Figure 4 with the method of [10]. By changing constants $\alpha$ and $\theta$, we can change the dynamic characteristics of the closed-loop reference active control system because the region where eigenvalues of the matrix $A_{c}=A_{0}-B_{a} K_{a}$ lie in the complex plane is changed. For example, the larger the constant $\alpha>0$ constraining the real part of the closedloop pole, the larger the expected damping of each mode of vibration because the underdamped pole of the $i$ th mode of vibration is given as the follows:

$$
p_{i}=-\zeta_{i} \omega_{i} \pm j \sqrt{1-\zeta_{i}^{2}} \omega_{i}, \quad i=1, \ldots, n, \quad j=\sqrt{-1}
$$

where $p_{i}, \zeta_{i}$, and $\omega_{i}$ are the pole, the damping ratio, and the undamped natural frequency of the $i$ th mode of vibration, respectively. Moreover, from the expression in (22), $\alpha$ and $\theta>0$ also constrain the value of the (undamped) natural frequencies $\omega_{i}, i=1, \ldots, n$, of the reference active control system as the imaginary part of the pole.

The state-feedback gain matrix $K_{a}$ satisfying the above closed-loop pole constraints can be obtained with the LMI (Linear Matrix Inequality) based method [10]. All poles of the closed-loop reference active control system lie in the region $R$ if $X=X^{T}>0$ exists satisfying matrix inequalities as follows:

$$
\begin{aligned}
& A_{c} X+X A_{c}^{T}+2 \alpha X=\left(A_{0}-B_{a} K_{a}\right) X+X\left(A_{0}-B_{a} K_{a}\right)^{T}+2 \alpha X<0 . \\
& {\left[\begin{array}{ll}
\sin \theta\left(A_{c} X+X A_{c}^{T}\right) & \cos \theta\left(A_{c} X-X A_{c}^{T}\right) \\
\cos \theta\left(X A_{c}^{T}-A_{c} X\right) & \sin \theta\left(A_{c} X+X A_{c}^{T}\right)
\end{array}\right]} \\
& \quad=\left[\begin{array}{ll}
\sin \theta\left\{\left(A_{0}-B_{a} K_{a}\right) X+X\left(A_{0}-B_{a} K_{a}\right)^{T}\right\} & \cos \theta\left\{\left(A_{0}-B_{a} K_{a}\right) X-X\left(A_{0}-B_{a} K_{a}\right)^{T}\right\} \\
\cos \theta\left\{X\left(A_{0}-B_{a} K_{a}\right)^{T}-\left(A_{0}-B_{a} K_{a}\right) X\right\} & \sin \theta\left\{\left(A_{0}-B_{a} K_{a}\right) X+X\left(A_{0}-B_{a} K_{a}\right)^{T}\right\}
\end{array}\right]<0
\end{aligned}
$$

With the change of variable $W=K_{a} X$, (23) become LMIs on $X=X^{T}>0$ and $W$ given as follows:

$$
\begin{gathered}
A_{0} X+X A_{0}^{T}-B_{a} W-W^{T} B_{a}^{T}+2 \alpha X<0, \\
{\left[\begin{array}{cc}
\sin \theta\left(A_{0} X+X A_{0}^{T}-B_{a} W-W^{T} B_{a}^{T}\right) & \cos \theta\left(A_{0} X-X A_{0}^{T}+W^{T} B_{a}^{T}-B_{a} W\right) \\
\cos \theta\left(X A_{0}^{T}-A_{0} X+B_{a} W-W^{T} B_{a}^{T}\right) & \sin \theta\left(A_{0} X-B_{a} W+X A_{0}^{T}-W^{T} B_{a}^{T}\right)
\end{array}\right]<0 .}
\end{gathered}
$$

Once matrices $X=X^{T}$ and $W$ are found, the state-feedback gain matrix $K_{a}$ that places the closed-loop poles in the region $\mathrm{R}$ is given by

$$
K_{a}=W X^{-1}
$$

The reference active control law in the output emulation approach is designed with the LMI-based method shown above. We define $\alpha>0$ and $\theta>0$ as design parameters to change the characteristics of the closed-loop reference active control system.
For the specific earthquake disturbance $v_{k}, k=1, \ldots, n_{c}$, the characteristics of the reference active control law are adjusted so that the control performance of the semiactive control system based on the output emulation approach is optimized. Two design parameters, $\alpha$ and $\theta$, that shape the region $R$ are optimized so that the semiactive control system based on the output emulation approach has the best control performance for the earthquake disturbance $v_{k}$. The reference active control law for the semiactive control law $C_{k}$ is defined as follows:

$$
u_{k}^{\mathrm{ra}}(t)=-K_{k} x_{a}(t), \quad k=1, \ldots, n_{c}
$$


where $u_{k}^{\mathrm{ra}}(t)$ and $K_{k}, k=1, \ldots, n_{c}$, are the active control input of the reference active control system and the state-feedback gain matrix of the $k$ th semiactive control law $C_{k}$. The statefeedback gain matrix $K_{k}$ is obtained so that the semiactive control system with $P$ and $C_{k}$ in Figure 3 achieves the best control performance for the $k$ th earthquake disturbance $v_{k}$.

In the design of the reference active control law, the parameter $\alpha$ enables adjusting the damping characteristic of the reference active control system and the parameter $\theta$ indirectly assigns the mode frequency of the control system. The physical meaning of the two parameters in vibration control of structural systems is clear. Furthermore, the reference active control that satisfies the pole placement constraints is formulated as the LMI problem (in (24)) that is efficiently solvable [10].

In obtaining the predictive controlled output, it is possible to use the discrete-time representation of the structural systems. In the discrete-time setting, the required amount of computation to obtain the predicted controlled output becomes smaller than that of the continuous time setting adopted in the present study. However, when we assume that the reference active control system is designed under the discrete-time setting, it is difficult to define a few design parameters that can easily understand the control characteristics like the above continuous time case because the stable region becomes the inside of a unit circle in a complex plane. Furthermore, the method for obtaining the discrete-time reference active control law satisfying the damping and mode frequency constraints have not been established to date as far as the authors know. Therefore, in this study, we designed the control system under the continuous time setting which facilitates the design of the optimum reference active control law.

Remark 3. In obtaining the optimal reference semiactive control in (26) for the earthquake disturbance $v_{k}, k=$ $1, \ldots, n_{c}$, we can adopt any indices if they properly represent the performance on vibration suppression of the semiactive control system subject to the disturbance $v_{k}$, for example, the quadratic performance index employed in the LQ optimal control and the peak value of the power spectrum density of the specific output signal subject to the disturbance $v_{k}$. The optimal design parameters of the reference active control system, $\alpha$ and $\theta$, are obtained to optimize the performance index obtained with the structural response of the semiactive control system (subject to the disturbance $v_{k}$ ) based on the output emulation approach.

\subsection{Design of the Adaptive Scheduling Mechanism. Design} method for the adaptive scheduling mechanism of $n_{c}$ semiactive control laws $C_{k}, k=1, \ldots, n_{c}$, is described in the following.

The adaptive scheduling is performed by interpolating the reference active control law used in the $n_{c}$ semiactive control laws obtained in the previous section. For example, when $n_{c}=$ 2 , that is, we have two semiactive control laws $C_{1}$ and $C_{2}$, the interpolated reference active control input $u_{I}^{\text {ra }}(t)$ is defined as follows:

$$
\begin{aligned}
u_{I}^{\mathrm{ra}}(t) & =\beta_{1}(t) u_{1}^{\mathrm{ra}}(t)+\left\{1-\beta_{1}(t)\right\} u_{2}^{\mathrm{ra}}(t) \\
& =-\left[\beta_{1}(t) K_{1}+\left\{1-\beta_{1}(t)\right\} K_{2}\right] x_{a}(t),
\end{aligned}
$$

where $0 \leq \beta_{1}(t) \leq 1$ is the parameter for the interpolation. The number of the interpolation parameters is $n_{c}-1$. The adaptive scheduling mechanism $S$ in the block diagram in Figure 2 outputs the interpolation parameters $\beta_{l}(t), l=$ $1, \ldots, n_{c}-1$, with the information on the earthquake disturbance $v(t)$, the controlled output signal $z(t)$, and available other signals. The interpolated reference active control input $u_{I}^{\mathrm{ra}}(t)$ is a convex combination of the $n_{c}$ reference active control input $u_{k}^{\mathrm{ra}}(t), k=1, \ldots, n_{c}$. In this study, with the interpolated reference active control $u_{I}^{\mathrm{ra}}(t)$, the command signal of the semiactive control device denoted by $u^{c}$ in Figure 2 is obtained with the output emulation approach described in the previous subsection.

Recall that each of the input signals of the reference active control system denoted by $u_{k}^{\mathrm{ra}}(t), k=1, \ldots, n_{c}$, is obtained to achieve the best control performance only for the $k$ th earthquake disturbance. For the earthquake disturbance (with unknown time and frequency characteristics) that occurs in the future, we can expect that the semiactive control law based on the interpolated reference active control input shows the good control performance if the semiactive control laws $\mathrm{C}_{k}, k=1, \ldots, n_{c}$, are designed for the recorded and/or artificial earthquake disturbances with a wide variety of the time and frequency characteristics and the interpolation parameters $0 \leq \beta_{l}(t) \leq 1, l=1, \ldots, n_{c}-1$, are properly tuned in real time. Then, we can escape from the control specification on the robust performance that can be the constraint in improving the control performance as discussed earlier when we adopt a single semiactive control law.

By optimizing design parameters $\alpha$ and $\theta$ under the semiactive control based on the output emulation approach in the previous subsection, it is possible to obtain a semiactive control law $C_{k}$ with good control performance for a single earthquake disturbance $v_{k}$. In fact, since there are only two design parameters, it is possible to apply an exhaustive search and find the globally optimal solution. Therefore, the remaining problem is to get a tuning strategy that interpolates $n_{c}$ reference active control laws with available information. In other words, we need to get a map $M$ from the available sensor data (and/or their functions) to the interpolation parameter $0 \leq \beta_{l}(t) \leq 1, l=1, \ldots, n_{c}-1$. If we get a good map $M$ to achieve high performance for a wide range of earthquake disturbances, we can adapt to the future occurring earthquake with unknown time and frequency characteristics. Such adaptive semiactive control law without constraints on the robust performance realizes further performance improvement.

However, at the moment, there is no clue to get a good map M. For example, we cannot determine what kind of sensor data should be used as input for $\mathrm{M}$ and what function should be employed as the map $M$ because we cannot clarify the relationship between the characteristics of the earthquake disturbance and that of the optimized semiactive control law. 


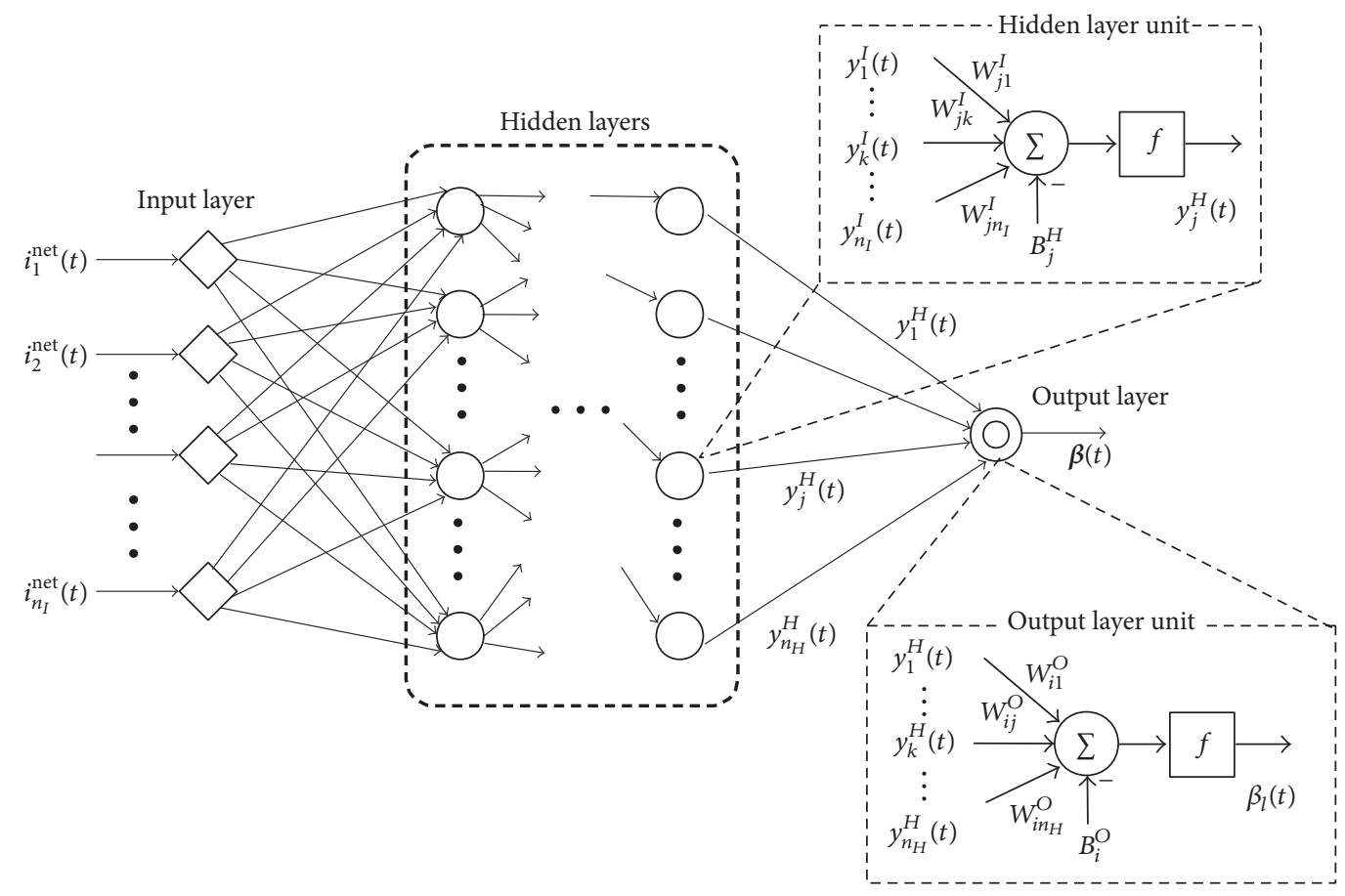

FIGURE 5: Structure of the multilayered ANN.

Therefore, in this study, we adopt a multilayered artificial neural network (ANN) as the basic structure of $M$ and optimizing ANN parameters according to the specification of semiactive control system. We use the ANN as the map M, expecting to have a high ability to construct various functions including nonlinear mappings [6].

A schematic diagram of the multilayered $\mathrm{ANN}$ is shown in Figure 5. The ANN input is a vector $\mathbf{i}^{\text {net }}(t)=$ $\left[i_{1}^{\text {net }}(t), \ldots, i_{n_{I}}^{\text {net }}(t)\right]^{T}$ which is composed of the available signal in the control system. Signals such as $y(t), u(t)$, and $v(t)$ of the structural model obtained by sensors installed on the control object are fed to the ANN input layer. The ANN outputs an interpolation parameter vector $\beta(t)=\left[\beta_{1}(t), \ldots, \beta_{n_{c}-1}\right]^{T}$ from the output layer. Elements of the vector $\beta(t)$ are the interpolation parameters of the reference active control input $0 \leq \beta_{l}(t) \leq 1, l=1, \ldots, n_{c}-1$. There are several hidden layers between the input and output layers to enhance the mapping description capability of the ANN.

To describe the structure of the ANN more specifically, let $U_{k}^{I}, k=1, \ldots, n_{I}, U_{j}^{H}, j=1, \ldots, n_{H}$, and $U_{i}^{O}, i=1, \ldots, n_{O}$, be units in the input, hidden, and output layers, respectively, where $n_{I}, n_{H}$, and $n_{O}=n_{c}-1$ are the number of neurons of the respective layers. Without loss of generality, we assume here that the number of the hidden layers is one for simplicity. The $l$ th element of the output layer of the network $\beta_{l}(t), l=$ $1, \ldots, n_{c}-1$, is obtained as follows:

$$
\beta_{l}(t)=f\left(\sum_{j=1}^{n_{h}} W_{l j}^{o} y_{j}^{h}(t)-B_{l}^{o}\right), \quad l=1, \ldots, n_{c}-1
$$

$$
\begin{aligned}
& y_{j}^{h}(t)=f\left(\sum_{k=1}^{n_{I}} W_{j k}^{H} y_{k}^{I}(t)-B_{j}^{H}\right) \quad j=1, \ldots, n_{H}, \\
& y_{k}^{I}(t)=\mathbf{i}_{k}^{\text {net }}(t), \quad k=1, \ldots, n_{I},
\end{aligned}
$$

where $W_{i j}^{O} \leq W_{i j}^{O} \leq \overline{W_{i j}^{O}}, W_{j k}^{H} \leq W_{j k}^{H} \leq \overline{W_{j k}^{H}}, \underline{B_{i}^{O}} \leq B_{i}^{O} \leq \overline{B_{i}^{O}}$, and $B_{j}^{H} \leq B_{j}^{H} \leq \overline{B_{j}^{H}}, i=1, \ldots, n_{O}, j=1, \ldots, n_{H}, k=1, \ldots, n_{I}$, are weights connecting $U_{i}^{O}$ and $U_{j}^{H}, U_{j}^{H}$ and $U_{k}^{I}$, and biases for $U_{i}^{O}$ and $U_{j}^{H}$, respectively. The function $f(x)$ is the sigmoid function given as

$$
f(x)=\frac{1}{1+e^{-x}} .
$$

Note that $0 \leq f(x) \leq 1 \forall x$. Because of $0 \leq f(x) \leq 1$, all the elements of the interpolation parameter $\beta_{l}(t), l=1, \ldots, n_{c}-1$ stay in the interval $0 \leq \beta_{l}(t) \leq 1, l=1, \ldots, n_{c}-1$. Design parameters of the ANN are the weights and bias in all the units of the ANN. These parameters are optimized as the adaptive scheduling mechanism to improve the control performance of the semiactive control system.

If the ideal interpolation parameters of the adaptive gain scheduling mechanism are available as train sets and they can be used for the ANN learning, we can use the back propagation method [11] to optimize the weights and biases of the ANN efficiently. However, the desired map denoted by $M_{d}$ is not known in advance under this problem formulation. That is, we do not know what is the best strategy to change the interpolation parameter $0 \leq \beta_{l} \leq 1, l=$ 
$1, \ldots, n_{c}-1$, for an earthquake disturbance at a certain time $t$. Therefore, in the present study, a genetic algorithm (GA) is applied to the optimization of the design parameters of the ANN. In the GA, all ANN design parameters are combined into one chromosome. Individuals with different chromosomes are randomly generated in the initial stage and the optimal chromosome is searched by the GA. In the GA search, genetic operations, for example, crossover, mutation, and selection, are repeatedly applied to all individuals so that the fitness of the individual is maximized; that is, the objective function representing the control performance of the semiactive control systems is optimized.

\section{Design Example}

A 6-DOF base-isolated structural system with a semiactive damper $[12,13]$ in Figure 6 is used as a design example. The structural parameters of the 6-DOF structural system are shown in Table 1 . The maximum and minimum damping coefficients of the semiactive damper in (1) are defined as $\overline{d_{1}^{s}}=20 \times 10^{3}[\mathrm{Ns} / \mathrm{m}], d_{1}^{s}=0[\mathrm{Ns} / \mathrm{m}]$ in (5), respectively.

In this example we obtain three semiactive control laws $C_{k}, k=1, \ldots, 3$, for the specific earthquake disturbance. These semiactive control laws are designed to exert the best control performance for three recorded earthquake disturbances used, that is, $k=1$ : El Centero NS (1940), $k=2$ : Hachinohe NS (1968), and $k=3\left(=n_{c}\right)$ : Kobe NS (1995) waves. The semiactive control law $C_{k}, k=1,2,3$, is obtained with the output emulation approach in Section 3.2 so that the control performance of the $k$ th semiactive control law is optimized for the corresponding $k$ th earthquake disturbance under the setting of the control system in Figure 3. The index for evaluating the control performance of the semiactive control system is defined as follows:

$$
\begin{aligned}
J_{k}= & \max \left(J_{k}^{s / \text { on }}, J_{k}^{s / \mathrm{off}}\right), \quad k=1, \ldots, n_{c} \\
J_{k}^{s / \mathrm{off}}= & \sum_{j=1}^{n} \frac{\operatorname{RMS}\left({ }^{k} r_{j}^{\text {semi }}\right)}{\operatorname{RMS}\left({ }^{k} r_{j}^{\text {off }}\right)}+\sum_{j=1}^{n} \frac{\operatorname{Peak}\left({ }^{k} r_{j}^{\text {semi }}\right)}{\operatorname{Peak}\left(k r_{j}^{\text {off }}\right)} \\
& +\sum_{j=1}^{n} \frac{\operatorname{RMS}\left({ }^{k} \ddot{q}_{j}^{\text {semi }}\right)}{\operatorname{RMS}\left({ }^{k} \ddot{q}_{j}^{\text {off }}\right)}+\sum_{j=1}^{n} \frac{\operatorname{Peak}\left({ }^{k} \ddot{q}_{j}^{\text {semi }}\right)}{\operatorname{Peak}\left({ }^{k} \ddot{q}_{j}^{\text {off }}\right)}, \\
J_{k}^{s / \text { on }}= & \sum_{j=1}^{n} \frac{\operatorname{RMS}\left({ }^{k} r_{k}^{\text {semi }}\right)}{\operatorname{RMS}\left({ }^{k} r_{j}^{\text {on }}\right)}+\sum_{j=1}^{n} \frac{\operatorname{Peak}\left(k r_{j}^{\text {semi }}\right)}{\operatorname{Peak}\left({ }^{k} r_{j}^{\text {on }}\right)} \\
& +\sum_{j=1}^{n} \frac{\operatorname{RMS}\left({ }^{k} \ddot{q}_{j}^{\text {semi }}\right)}{\operatorname{RMS}\left({ }^{k} \ddot{q}_{j}^{\text {on }}\right)}+\sum_{j=1}^{n} \frac{\operatorname{Peak}\left({ }^{k} \ddot{q}_{j}^{\text {semi }}\right)}{\operatorname{Peak}\left(k \ddot{q}_{j}^{\text {on }}\right)},
\end{aligned}
$$

where ${ }^{k} r_{j}^{*}$ and ${ }^{k} \ddot{q}_{j}^{*}, j=1, \ldots, n, *=$ semi, off, or on are the relative displacement between $j$ and $(j-1)$-th floors and the absolute acceleration of the $j$ th floor for the $k \mathrm{th}, k=1, \ldots, n_{c}$, earthquake disturbance, respectively. Superscripts $*=$ semi, off, or on are the case of the semiactive control based on the proposed output emulation approach, passive off, and passive

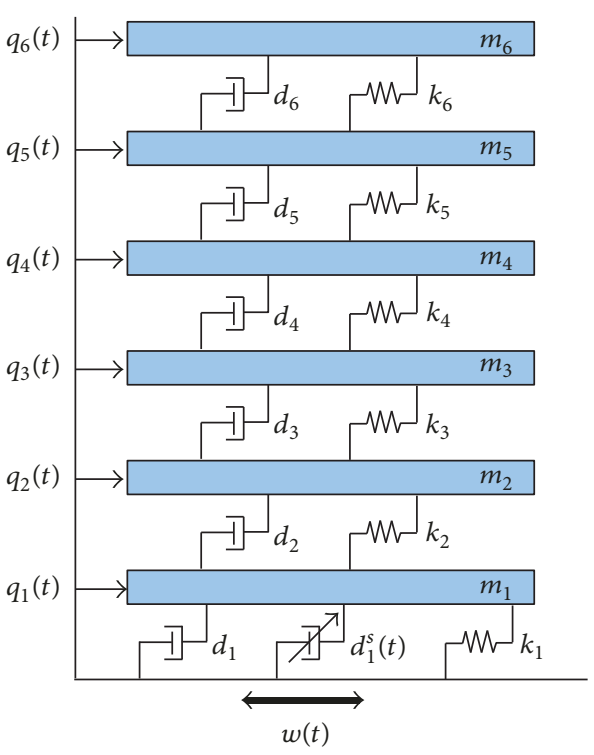

FIGURE 6: 6-DOF base-isolated structural system.

TABLE 1: Structural parameters of the 6-DOF structural system.

\begin{tabular}{lccc}
\hline Floor & $m_{i}[\mathrm{~kg}]$ & $d_{i}[\mathrm{kNs} / \mathrm{m}]$ & $k_{i}[\mathrm{kN}]$ \\
\hline 1 & 6800 & 7.45 & 231.50 \\
2 & 5897 & 670 & 33732 \\
3 & 5897 & 580 & 29093 \\
4 & 5897 & 570 & 28621 \\
5 & 5897 & 500 & 24954 \\
6 & 5897 & 380 & 19059 \\
\hline
\end{tabular}

TABle 2: Optimal design parameters of the semiactive control for each earthquake disturbance.

\begin{tabular}{lcc}
\hline Earthquake wave $(k)$ & $\alpha$ & $\theta(\times \pi / 2)$ \\
\hline El Centro NS $(k=1)$ & 0.20 & 0.85 \\
Hachinohe NS $(k=2)$ & 0.38 & 1 \\
Kobe NS $(k=3)$ & 0.20 & 0.92 \\
\hline
\end{tabular}

on cases, respectively. Indices $J_{k}^{s / \text { off }}$ and $J_{k}^{s / \text { on }}$ are functions to evaluate the control performance of the semiactive control system in the case of the $k$ th earthquake disturbance, that is, $J_{k}^{s / \text { off }}$ : the semiactive control case compared to the passive off case, $J_{k}^{s / o n}$ : the semiactive control case compared to the passive on case.

The optimal design parameters for each earthquake disturbance are summarized in Table 2. These optimal design parameters are obtained with the exhaustive search in a range $0.1 \leq \alpha \leq 0.4$ and $0.7 \times(\pi / 2) \leq \beta \leq 1 \times(\pi / 2)$. Since the optimal design parameters for the specified earthquake disturbance are different from each other, the semiactive control based on the optimal reference active control law for a specific earthquake disturbance has different control characteristics. 

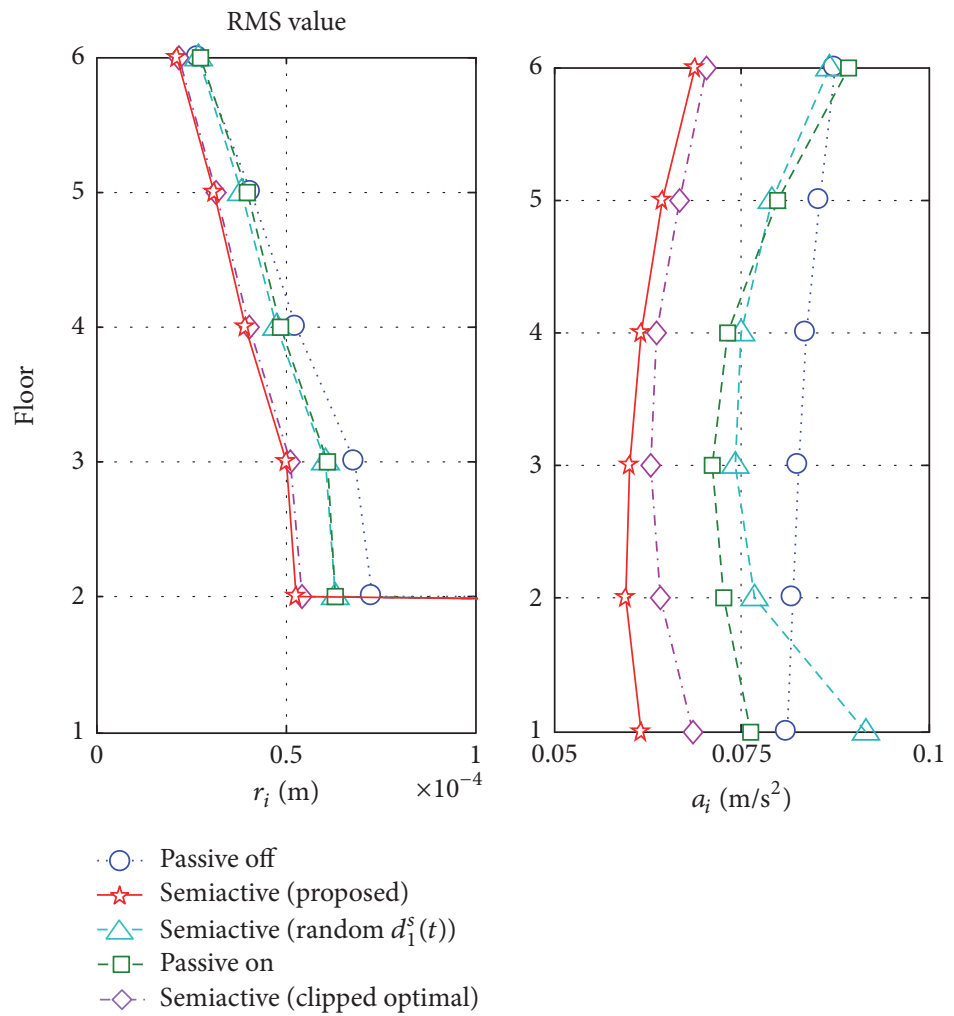

Figure 7: Simulation results for the El Centro NS (1940) earthquake disturbance; RMS values of the relative displacement between neighboring floors and absolute acceleration of each floor.

As the adaptive scheduling mechanism, the ANN with two output signals $0 \leq \beta_{l} \leq 1, l=1,2$, for the interpolation of three reference active control laws corresponds to three semiactive control laws $C_{k}, k=1,2,3$. The interpolated reference active control input denoted by $u_{I}^{\mathrm{ra}}(t)$ is the convex combination of $u_{k}^{\mathrm{ra}}(t), k=1,2,3$ with $\beta_{1}(t)$, and $\beta_{2}(t)$ given by

$$
\begin{aligned}
u_{I}^{\mathrm{ra}} & (t)=\left\{1-\beta_{1}(t)\right\} u_{1}^{\mathrm{ra}}(t)+\beta_{1}\left\{1-\beta_{2}(t)\right\} u_{2}^{\mathrm{ra}}(t) \\
& +\beta_{1}(t) \beta_{2}(t) u_{3}^{\mathrm{ra}}(t) \\
= & {\left[\left\{1-\beta_{1}(t)\right\} K_{1}+\beta_{1}\left\{1-\beta_{2}(t)\right\} K_{2}\right.} \\
& \left.+\beta_{1}(t) \beta_{2}(t) K_{3}\right] x_{a}(t),
\end{aligned}
$$

where $K_{k}, k=1,2,3$, is the state-feedback gain matrix for the $k$ th earthquake disturbance obtained each of which is obtained with optimal design parameters in Table 2.

As the input signal of the ANN, we employ the acceleration of the earthquake wave $\ddot{w}(t)$, its time derivative, and integral. Namely, the input signal vector $\mathbf{i}^{\text {net }}$ is given as

$$
\mathbf{i}^{\text {net }}=\left[\begin{array}{c}
\ddot{w}(t) \\
\dot{w}(t) \\
\frac{d \ddot{w}(t)}{d t}
\end{array}\right] .
$$

In the design example, we introduce two hidden layers whose numbers of units are 40 and 5, respectively. The number of units in each hidden layer is determined by trial and error. The total number of design parameters in the ANN becomes 377 and they are optimized with the GA. The objective function to evaluate the control performance of the semiactive control system is defined as follows:

$$
J=\sum_{i=1}^{n_{w}} J_{i}
$$

where $n_{w}$ is the number of earthquake disturbances to obtain the simulated structural response for the GA-based optimization. The index $J_{i}, i=1, \ldots, n_{w}$, is the performance index defined in (30) for the $i$ th earthquake disturbance. In the GA-based optimization of the ANN, the objective function $J$ in (34) is obtained with the simulation for all individuals containing design parameter of the ANN with the above three earthquake disturbances and the BCJL1 (artificial) wave, that is, we determine $n_{w}=4$ in (34). We set the population (number of individuals) of each generation and the maximum number of the generations in the GA as 20 and 200, respectively.

The results of the optimized semiactive control system for El Centro NS, BCJL1, Hachinohe NS, and Kobe NS waves are shown in Figures 7, 8, 10, 11, 13, 14, 16, and 17, respectively. The result of the case where the variable damping coefficient 


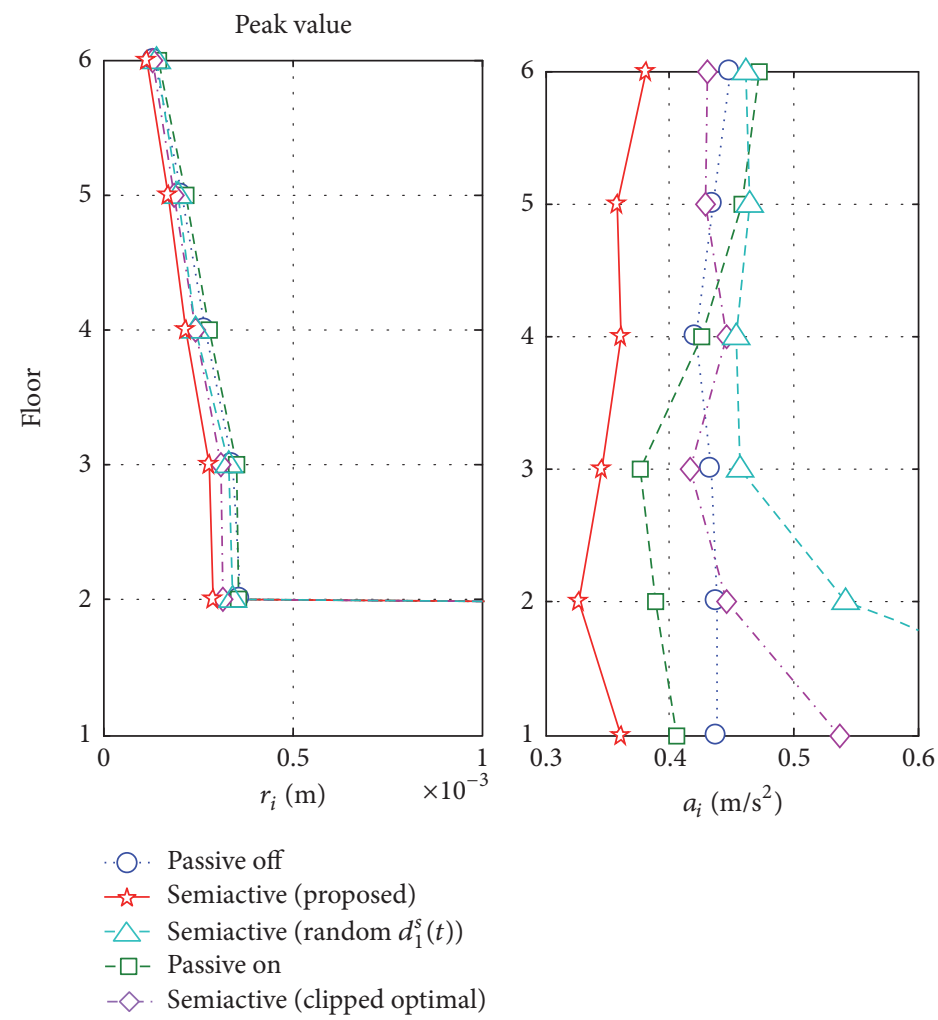

FIGURE 8: Simulation results for the El Centro NS (1940) earthquake disturbance; peak values of the relative displacement between neighboring floors and absolute acceleration of each floor.

$d_{1}^{s}(t)$ of the semiactive damper is randomly changed is also shown in the figures. The semiactive control based on the proposed approach shows good control performance for all the earthquake disturbances over the passive off and on and the random change cases.

Moreover, for comparison, the results of the case with the clipped optimal control [1], which is a popular and conventional semiactive control law that is often applied to building structures, are also shown in each figure. For the reference active control law of the clipped optimal control, the state-feedback control law in (10) is adopted as well as design parameters with the same pole location constraints as the present formulation. Design parameters $\alpha$ and $\theta$ minimizing the index in (34) are obtained by using the exhaustive search. In this case, the clipped optimal control law is designed to have robust performance characteristics, that is, to improve control performance on average for all considered earthquake disturbances. We can clearly see that the proposed semiactive control with the adaptive scheduling mechanism achieves better control performance compared with that of the clipped optimal control.

To see the behavior of the adaptive scheduling mechanism for each earthquake disturbance, the variable damping coefficient of the semiactive damper and the output of the ANN $\beta_{1}(t)$ and $\beta_{2}(t)$ are shown in Figures $9,12,15$, and 18 , respectively. It is found that the ANN generates the interpolation
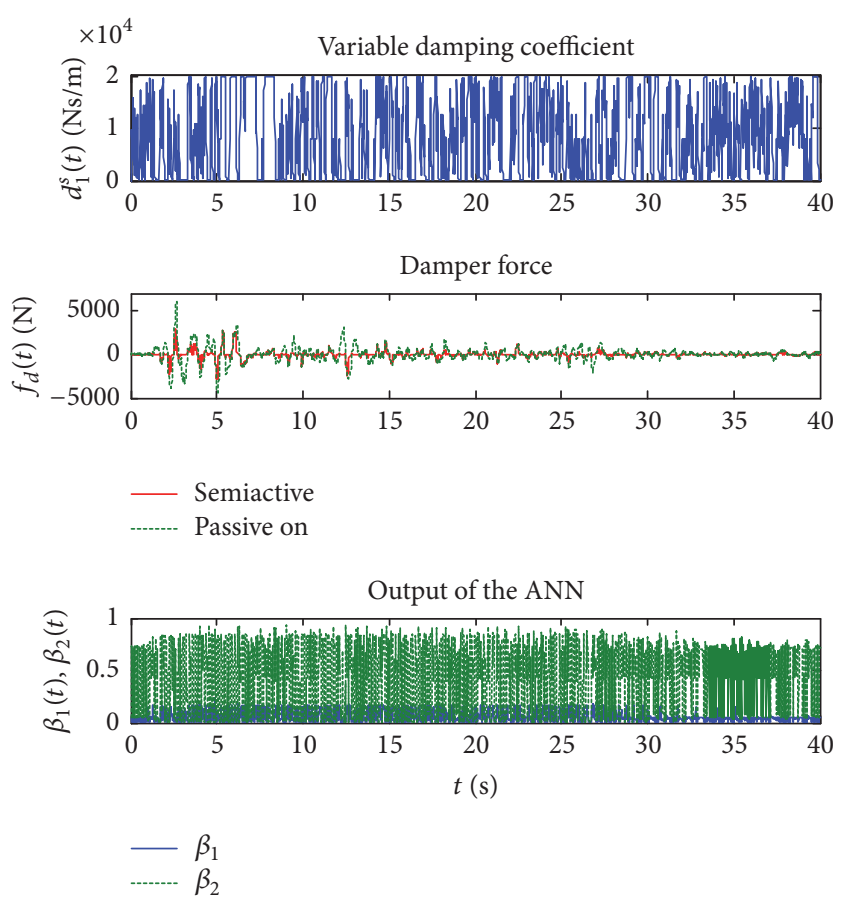

FIGURE 9: Simulation results for the El Centro NS (1940) earthquake disturbance; variable damping coefficient $d_{1}^{s}(t)$, force of damper $f_{d}(t)$, and output signals of the ANN $\beta_{1}(t)$ and $\beta_{2}(t)$. 


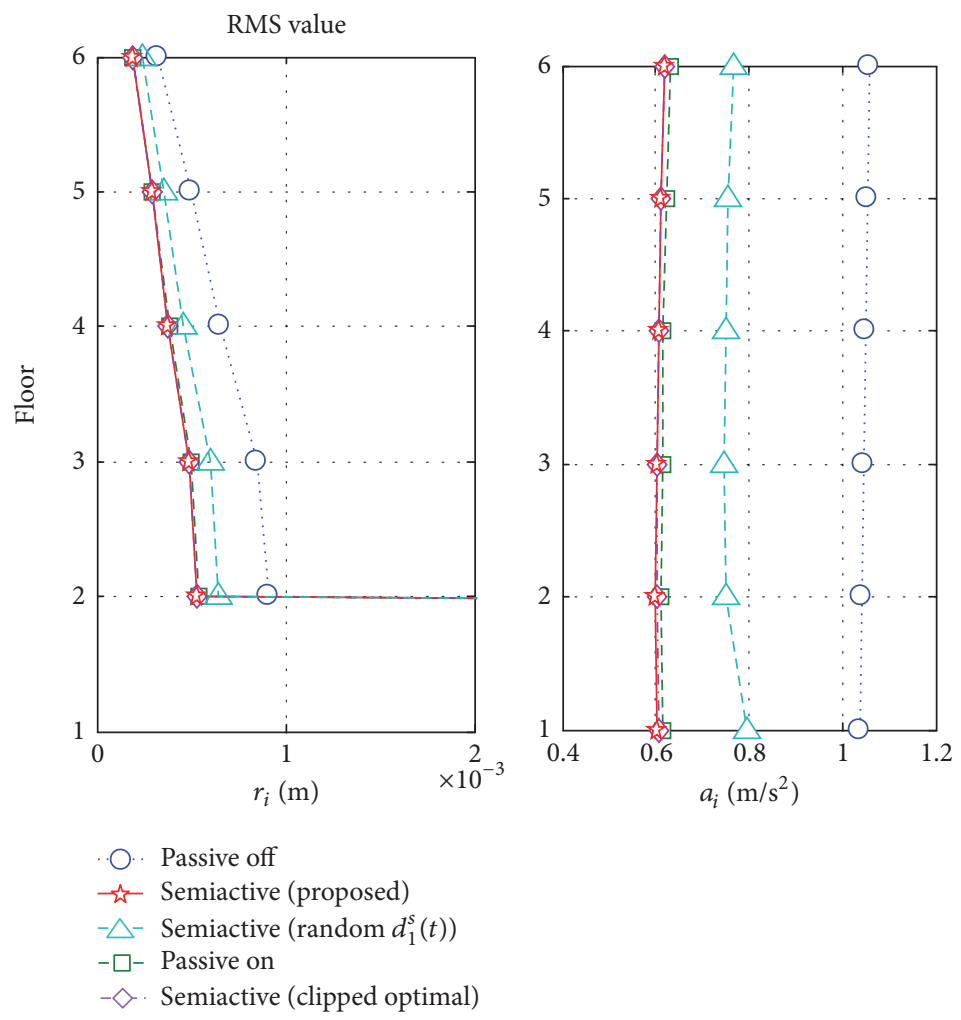

FIGURE 10: Simulation results for the BCJL1 earthquake disturbance; RMS values of the relative displacement between neighboring floors and absolute acceleration of each floor.
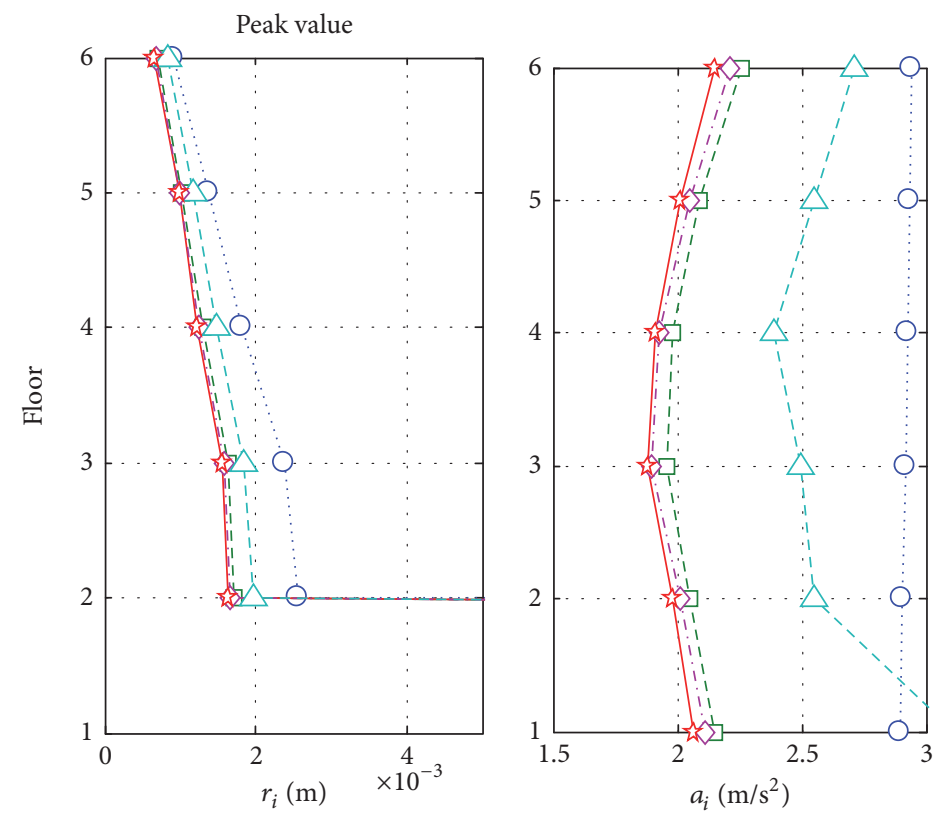

$\bigcirc$. Passive off
$-\downarrow-$ Semiactive (proposed)
$-\triangle-$ Semiactive (random $\left.d_{1}^{s}(t)\right)$
$-\square-$ Passive on
$-\diamond$. Semiactive (clipped optimal)

FIGURE 11: Simulation results for the BCJL1 earthquake disturbance; peak values of the relative displacement between neighboring floors and absolute acceleration of each floor. 

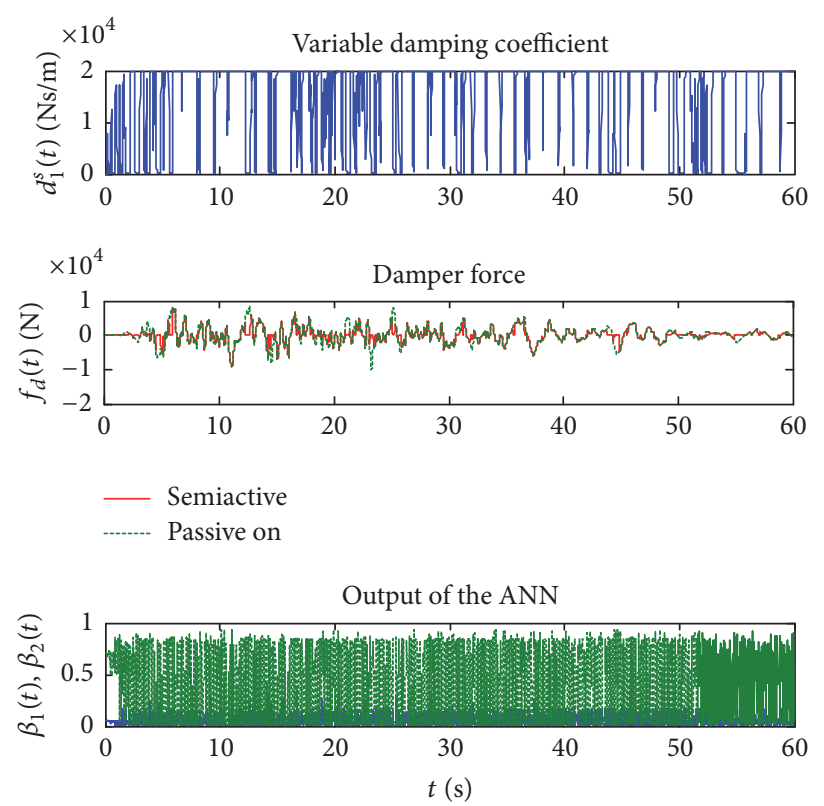

$$
\begin{array}{r}
-\beta_{1} \\
-\quad \beta_{2}
\end{array}
$$

FIGURE 12: Simulation results for the BCJL1 earthquake disturbance; variable damping coefficient $d_{1}^{s}(t)$, force of damper $f_{d}(t)$, and output signals of the ANN $\beta_{1}(t)$ and $\beta_{2}(t)$.
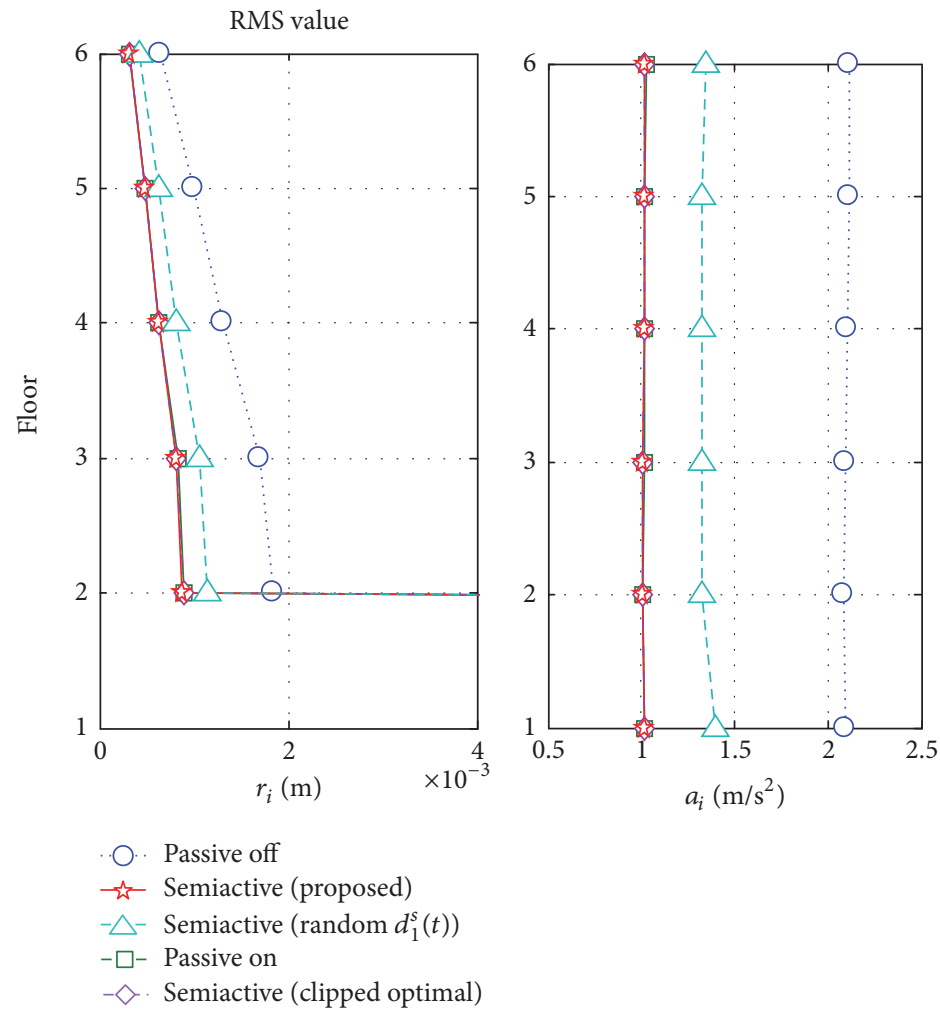

$$
\begin{aligned}
& -\checkmark \text { Semiactive (proposed) } \\
& \left.-\triangle-\text { Semiactive (random } d_{1}^{s}(t)\right) \\
& -\square-\text { Passive on } \\
& -\checkmark \text {. Semiactive (clipped optimal) }
\end{aligned}
$$

FIGURE 13: Simulation results for the Hachinohe NS (1968) earthquake disturbance; RMS values of the relative displacement between neighboring floors and absolute acceleration of each floor. 

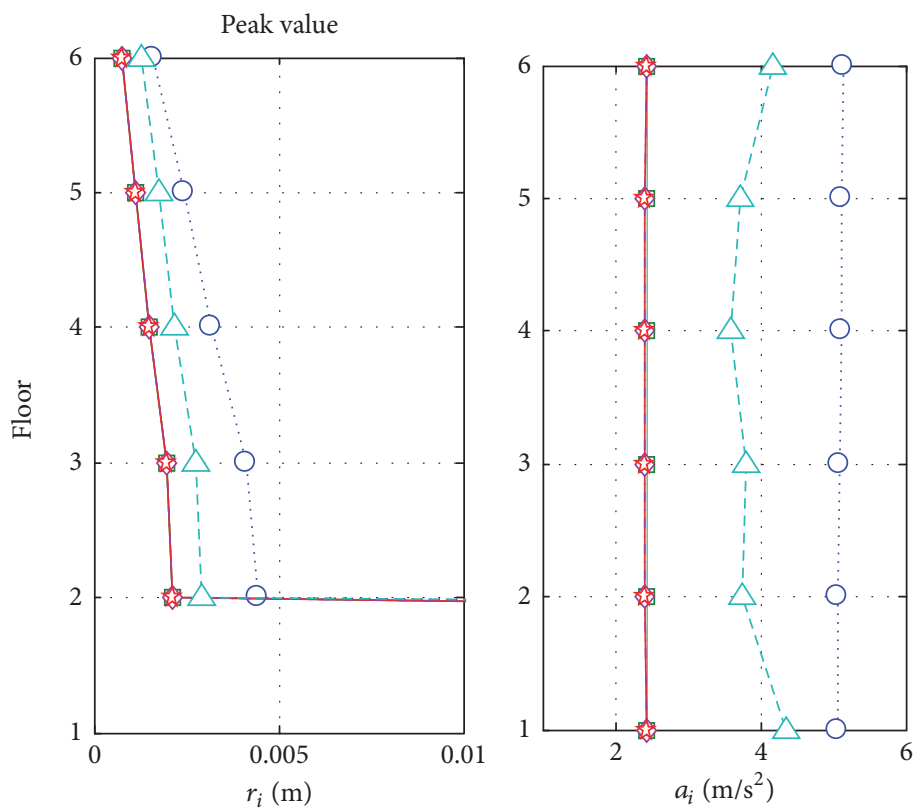

$$
\begin{aligned}
& \bigcirc . \text { Passive off } \\
& -\checkmark \text { Semiactive (proposed) } \\
& \left.-\triangle-\text { Semiactive (random } d_{1}^{s}(t)\right) \\
& -\square-\text { Passive on } \\
& -\diamond . \text { Semiactive (clipped optimal) }
\end{aligned}
$$

FIGURE 14: Simulation results for the Hachinohe NS (1968) earthquake disturbance; peak values of the relative displacement between neighboring floors and absolute acceleration of each floor.
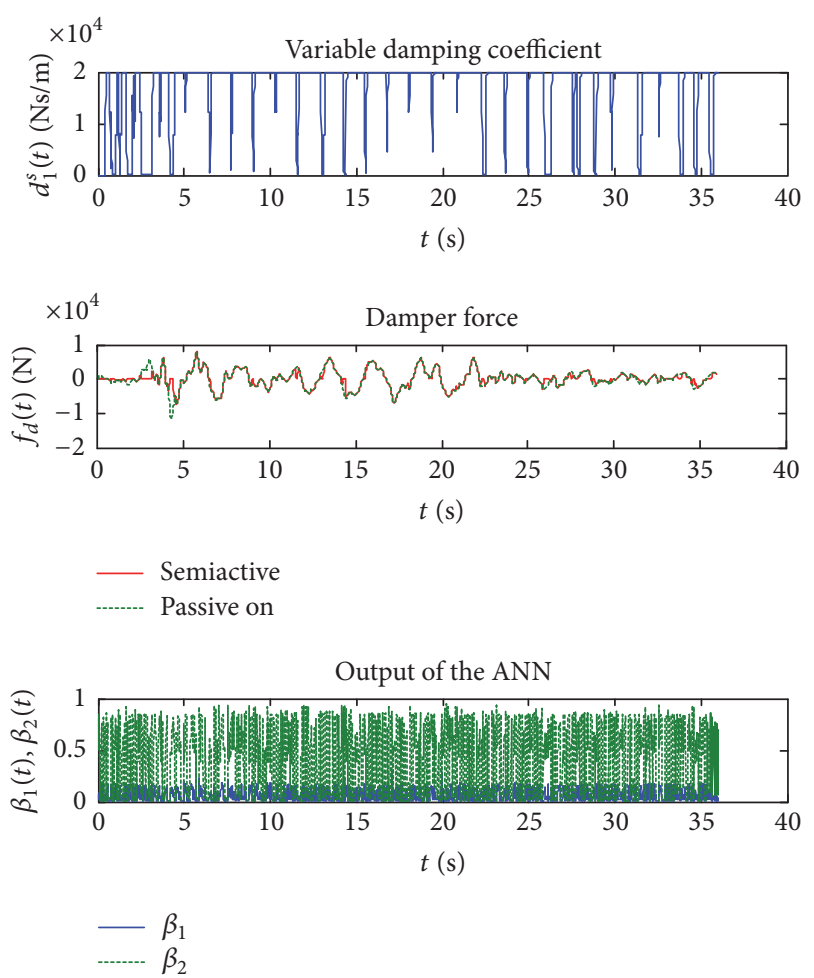

FIGURE 15: Simulation results for the Hachinohe NS (1968) earthquake disturbance; variable damping coefficient $d_{1}^{s}(t)$, force of damper $f_{d}(t)$, and output signals of the $\operatorname{ANN} \beta_{1}(t)$ and $\beta_{2}(t)$. parameters to optimize the control performance. The time history of the force of the semiactive damper $f_{d}(t)=$ $d_{1}^{s}(t)\left(\dot{q}_{1}(t)-\dot{w}(t)\right)$ for each earthquake disturbance with that in the passive on case is shown in those figures. Also, the peak value of the damper force in each case is in Table 3. We can clearly see that the force of the damper in the case of the semiactive control is much smaller than that of the passive on case. The result shows that the proposed semiactive control method shows good control performance on not only the vibration suppression but also the required damper force compared to the conventional passive control method.

To evaluate the control performance of the semiactive control system for the unknown earthquake disturbance, results for the Taft NS (1952) and Akita NS (1983) waves are shown in Figures 19, 20, 22, and 23, respectively. The variable damping coefficient of the semiactive damper, the force of the damper (in the semiactive and passive on cases), and the output of the ANN are also shown in Figures 21 and 24. Peak values of the damper force in the semiactive and passive on cases are shown in two rows at the bottom of Table 3. With the result, we can see that the good control performance is also achieved, compared to not only that of the passive off and passive on cases, but also the clipped optimal control case. This result shows that the proposed adaptive gain scheduled semiactive control method has superior control performance compared with the control method aimed at robust performance, which is conventionally used. 


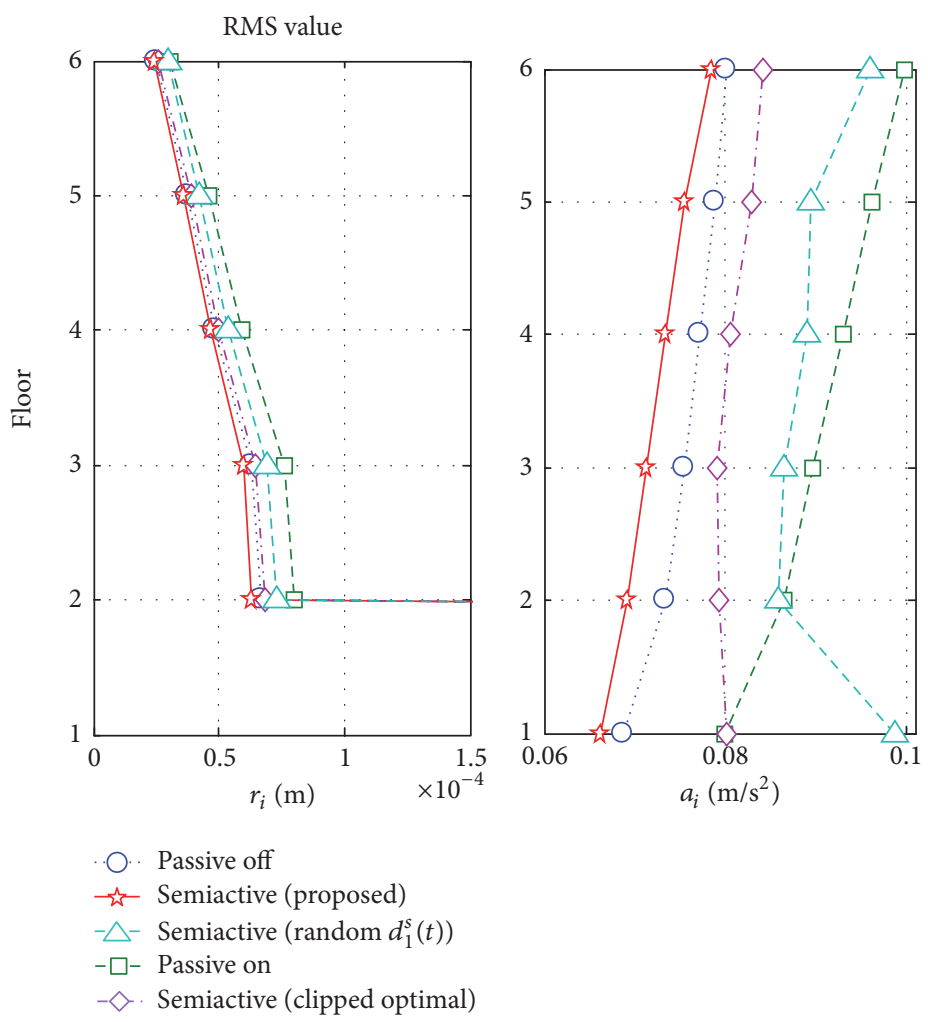

FIGURE 16: Simulation results for the Kobe NS (1995) earthquake disturbance; RMS values of the relative displacement between neighboring floors and absolute acceleration of each floor.

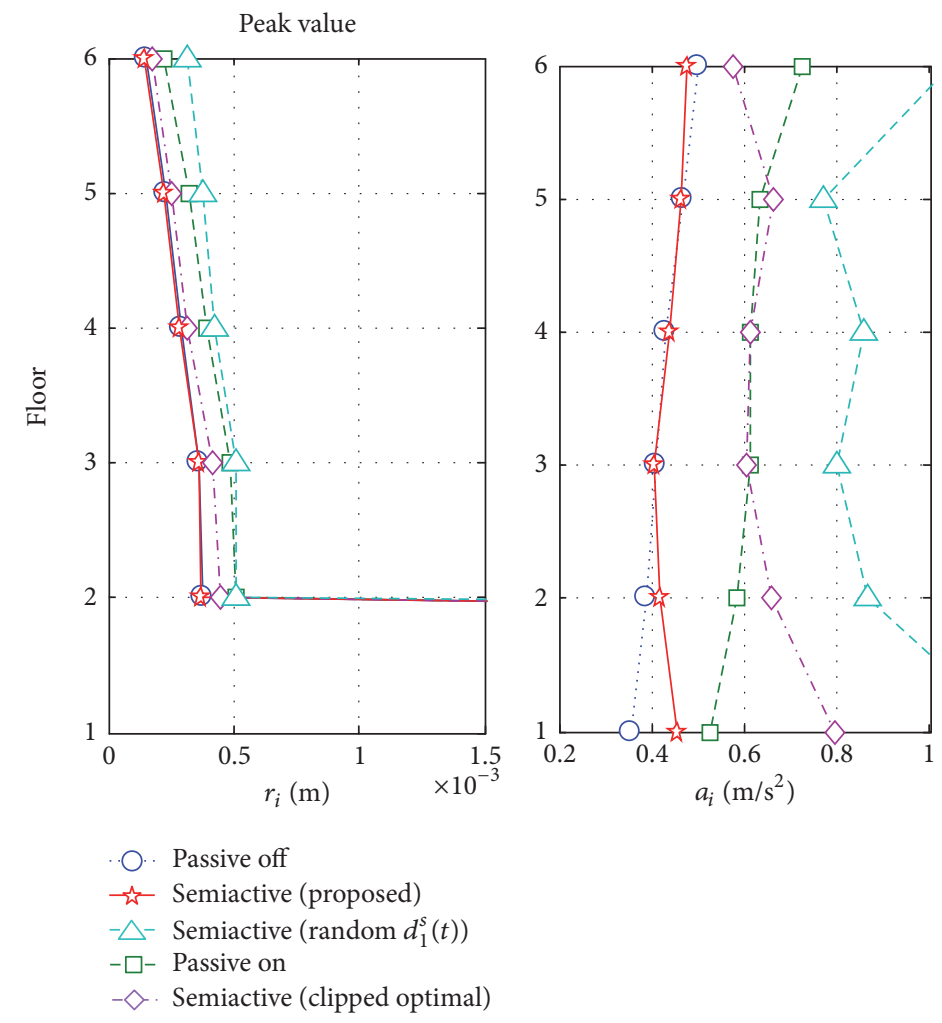

FIGURE 17: Simulation results for the Kobe NS (1995) earthquake disturbance; peak values of the relative displacement between neighboring floors and absolute acceleration of each floor. 

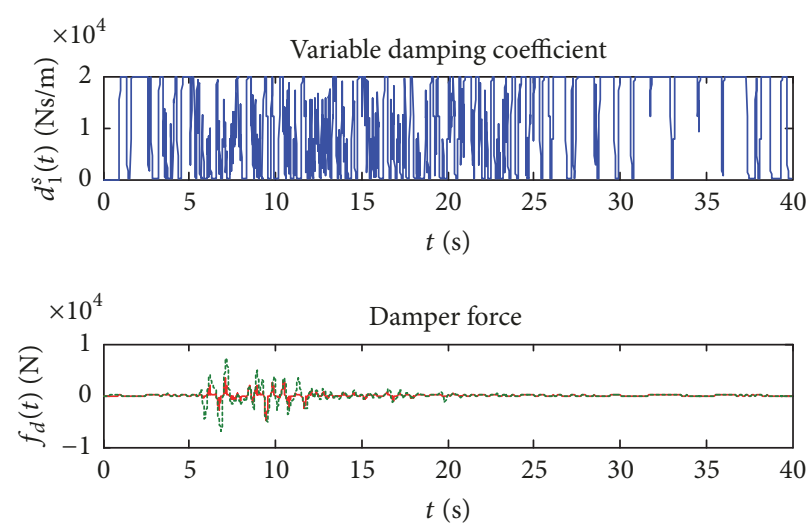

- Semiactive

-...-.- Passive on

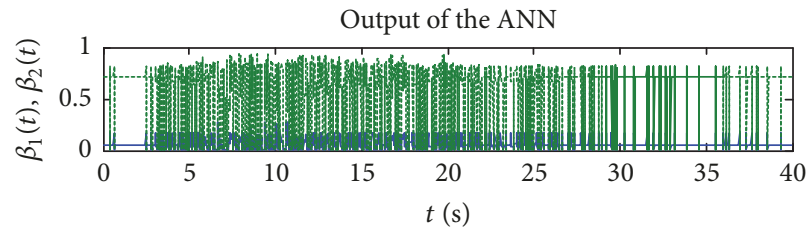

$-\beta_{1}$

FIGURE 18: Simulation results for the Kobe NS (1995) earthquake disturbance; variable damping coefficient $d_{1}^{s}(t)$, force of damper $f_{d}(t)$, and output signals of the ANN $\beta_{1}(t)$ and $\beta_{2}(t)$.
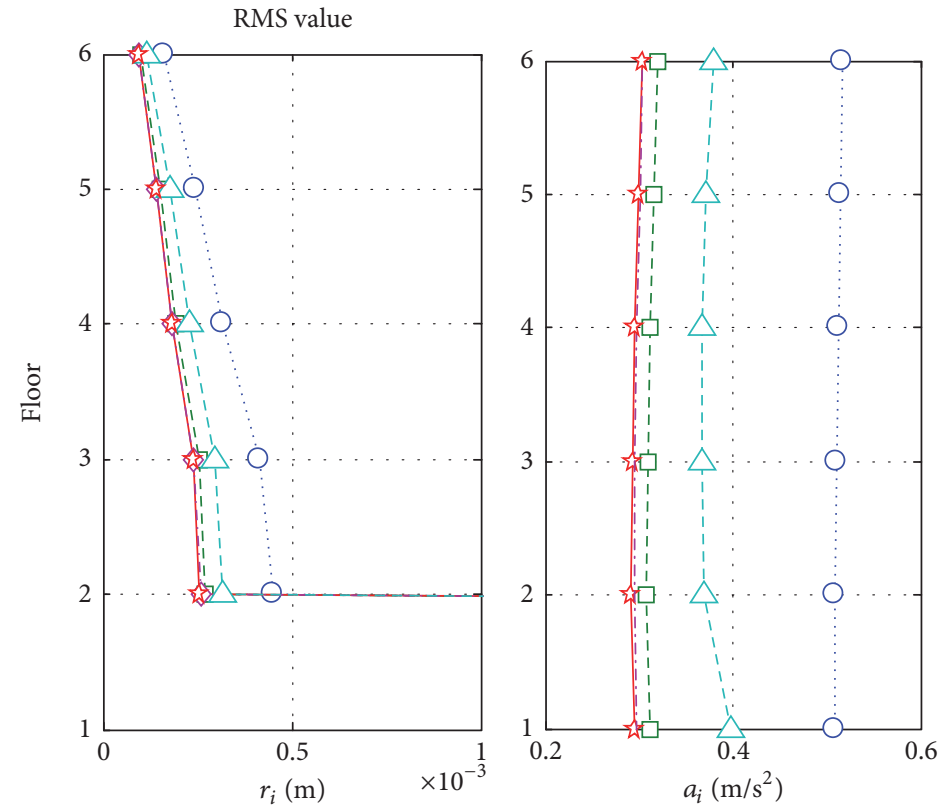

$$
\begin{aligned}
& \bigcirc . \text { Passive off } \\
& \checkmark-\text { Semiactive (proposed) } \\
& \left.-\triangle-\text { Semiactive (random } d_{1}^{s}(t)\right) \\
& -\square-\text { Passive on } \\
& -\triangleleft \text {. Semiactive (clipped optimal) }
\end{aligned}
$$

FIGURE 19: Simulation results for the Taft NS (1952) earthquake disturbance; RMS values of the relative displacement between neighboring floors and absolute acceleration of each floor. 


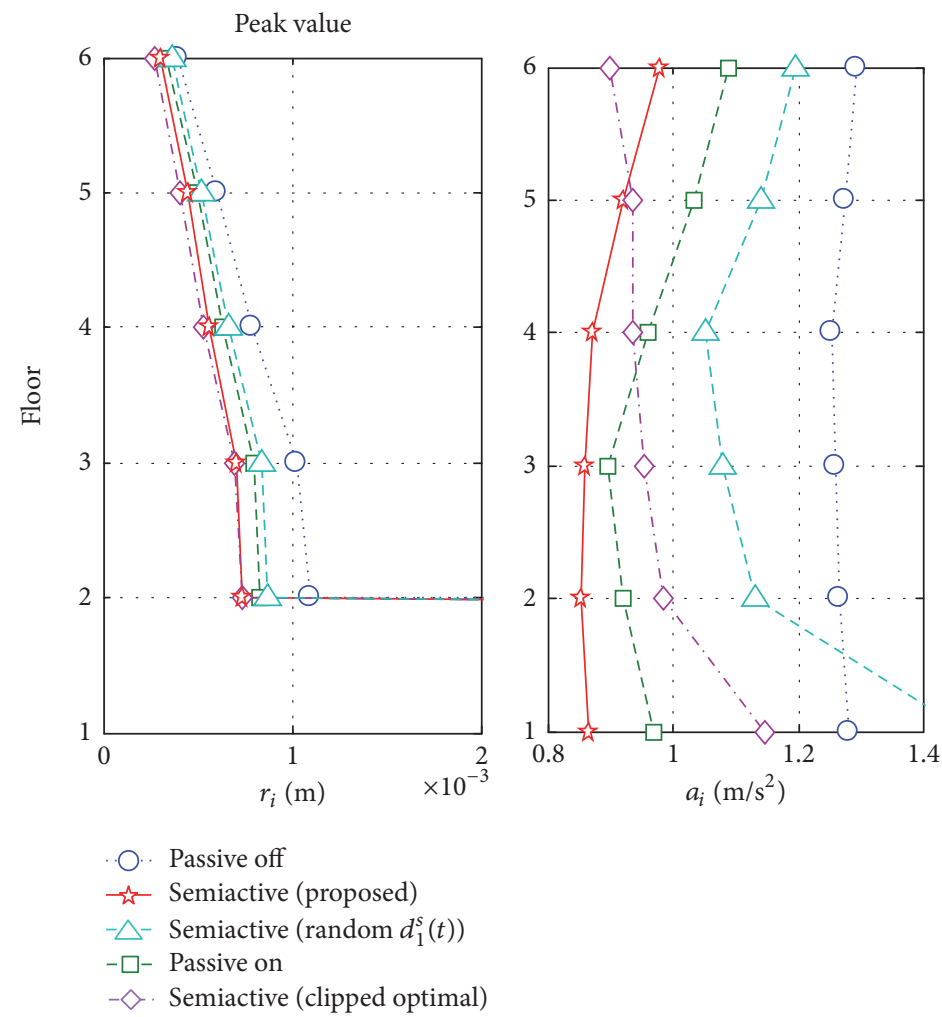

Figure 20: Simulation results for the Taft NS (1952) earthquake disturbance; Peak values of the relative displacement between neighboring floors and absolute acceleration of each floor.
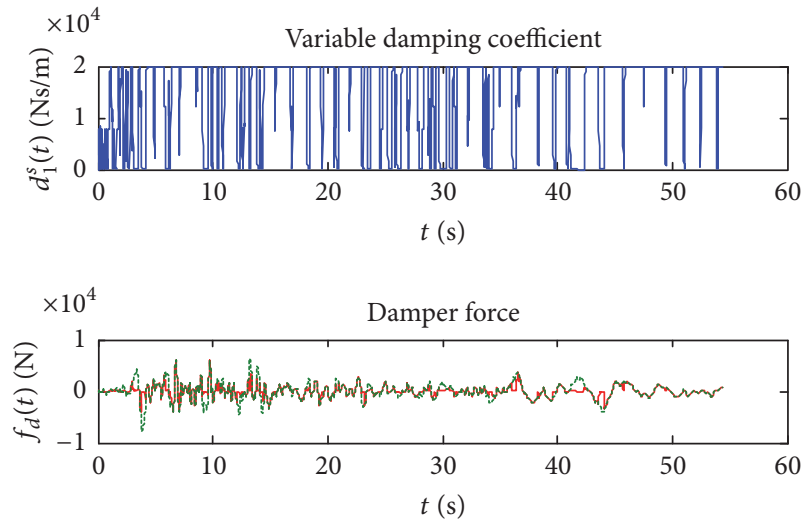

- Semiactive

-..-...- Passive on

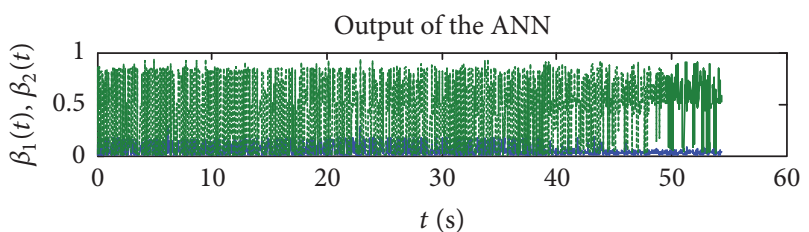

$\begin{aligned}- & \beta_{1} \\ -\cdots--. & \beta_{2}\end{aligned}$

FIGURE 21: Simulation results for the Taft NS (1952) earthquake disturbance; variable damping coefficient $d_{1}^{s}(t)$, force of damper $f_{d}(t)$, and output signals of the ANN $\beta_{1}(t)$ and $\beta_{2}(t)$.
TABle 3: Peak force of the damper.

\begin{tabular}{lcc}
\hline Earthquake & Passive on $[\mathrm{kN}]$ & Semiactive $[\mathrm{kN}]$ \\
\hline El Centro NS & 6.04 & 2.97 \\
BCJL1 & 10.02 & 9.24 \\
Hachinohe NS & 11.65 & 7.83 \\
Kobe NS & 7.29 & 5.00 \\
Taft NS & 7.85 & 6.02 \\
Akita NS & 9.39 & 8.20 \\
\hline
\end{tabular}

\section{Conclusion}

In order to achieve high control performance of semiactive vibration control, we have proposed a control strategy consisting of adaptive scheduling mechanisms of multiple semiactive control laws with specific control characteristics. The adaptive scheduling mechanism is designed as an interpolator of the reference active control laws, each of which is designed for a specific law for the single specified seismic wave and used for the semiactive control based on the output emulation approach. The ANN is adopted as the adaptive scheduling mechanism and design parameters of the ANN are optimized by the GA. Compared to the passive control case and the conventional semiactive control method based on the clipped optimal control method, the proposed semiactive control method is shown to have better control performance on vibration suppression and flexibility 


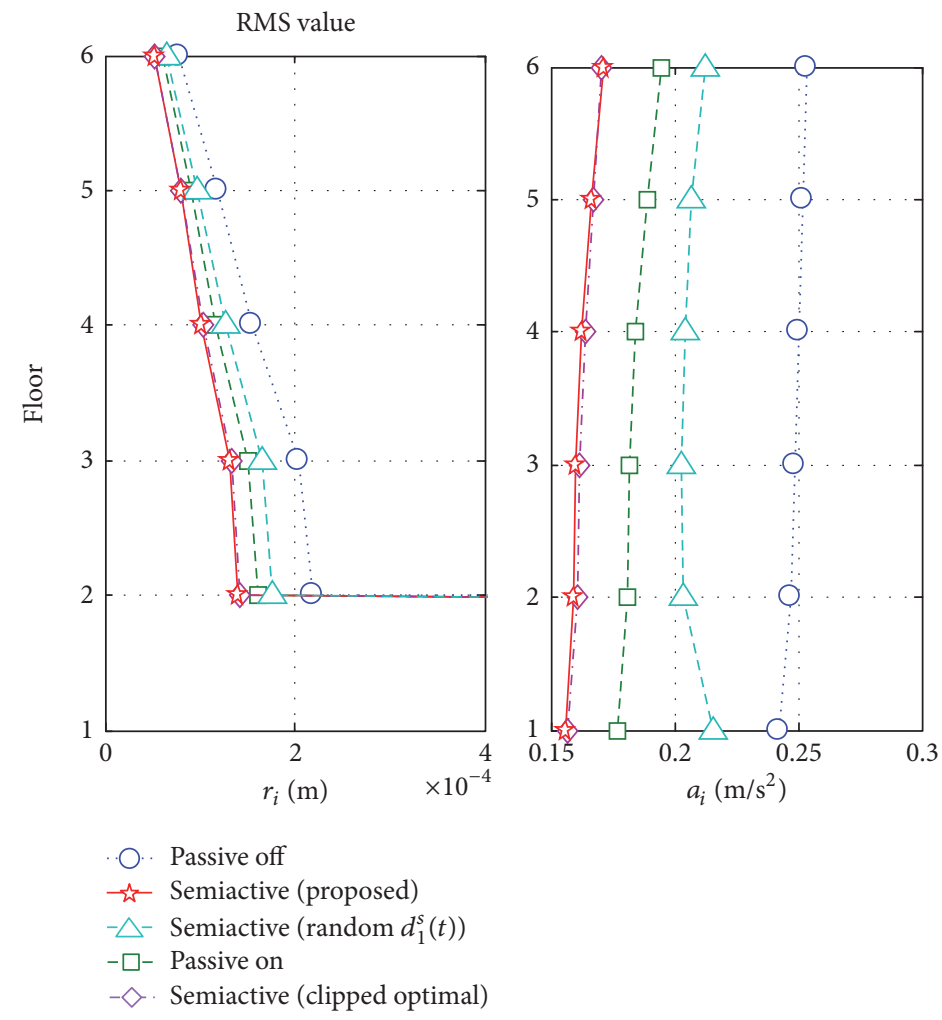

FIGURE 22: Simulation results for the Akita NS (1983) earthquake disturbance; RMS values of the relative displacement between neighboring floors and absolute acceleration of each floor.

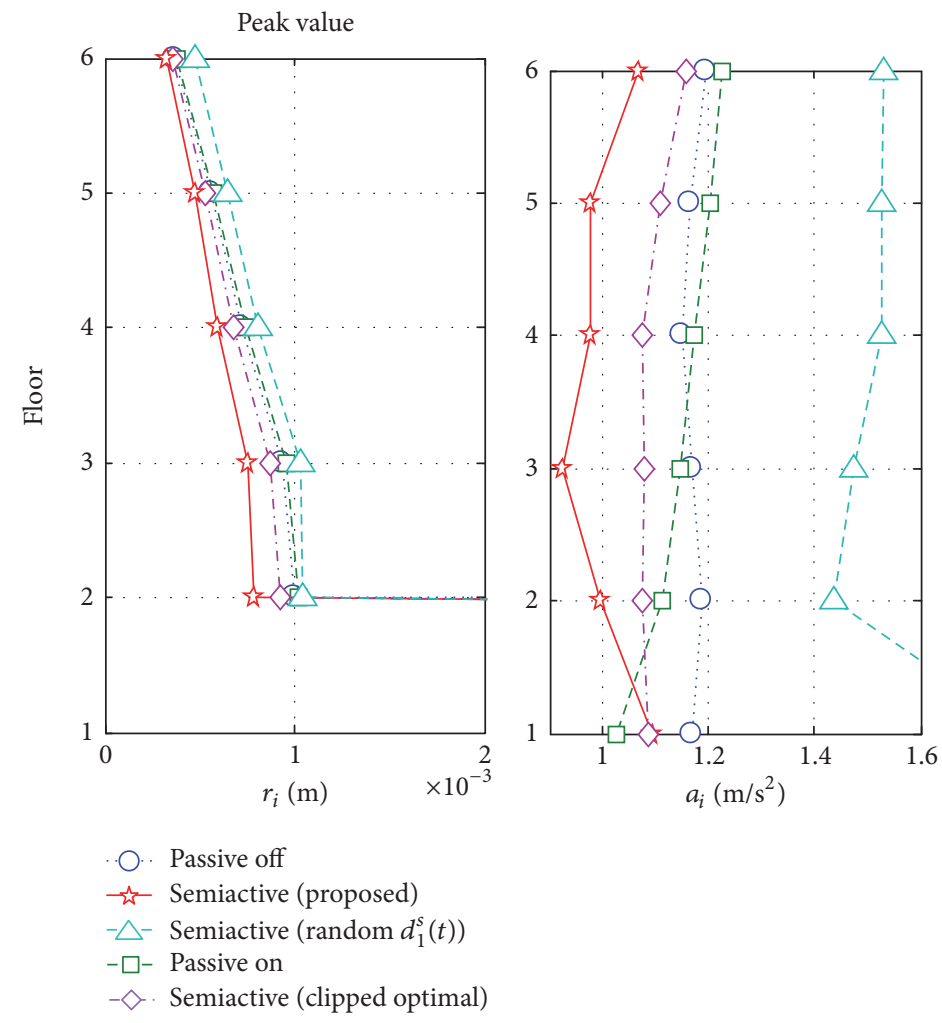

FIGURE 23: Simulation results for the Akita NS (1983) earthquake disturbance; peak values of the relative displacement between neighboring floors and absolute acceleration of each floor. 

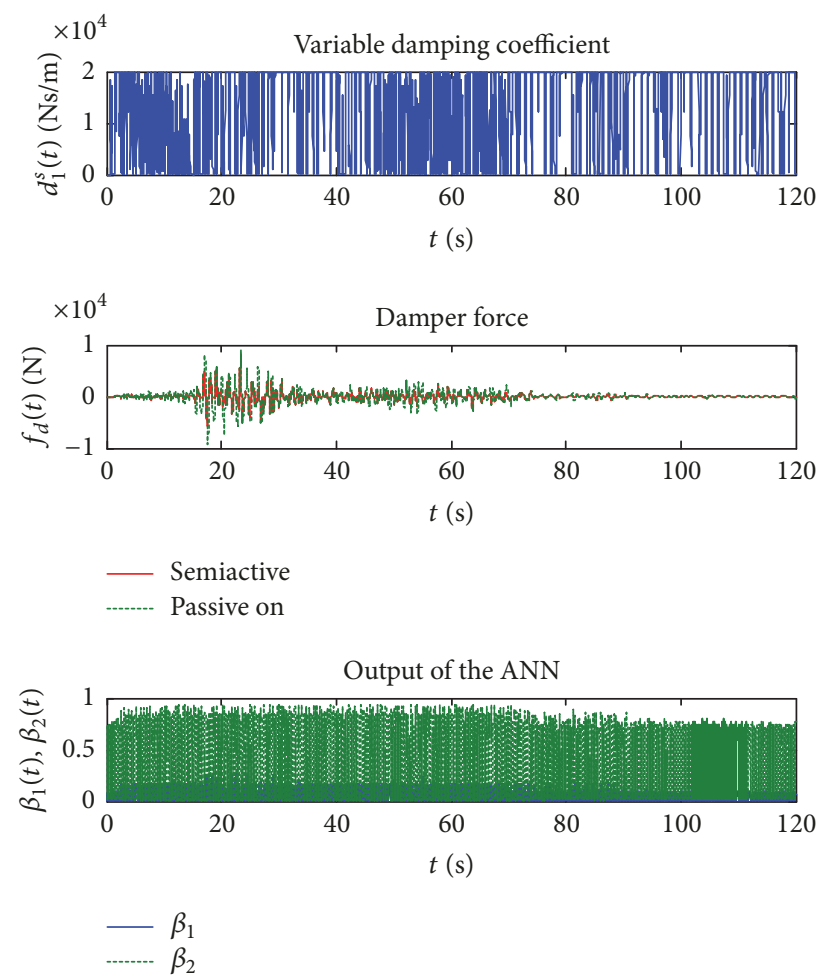

FIGURE 24: Simulation results for the Akita NS (1983) earthquake disturbance; variable damping coefficient $d_{1}^{s}(t)$, force of damper $f_{d}(t)$, and output signals of the ANN $\beta_{1}(t)$ and $\beta_{2}(t)$.

against earthquake disturbances whose time and frequency characteristics are unknown over the conventional semiactive control approach aiming at the robust performance characteristics.

\section{Conflicts of Interest}

The authors declare that they have no conflicts of interest.

\section{Acknowledgments}

This work was partially supported by Japan Society for the Promotion of Science (JSPS), Grant-in-Aid for Scientific Research (C), 15K05858, 2016.

\section{References}

[1] S. J. Dyke, B. F. Spencer Jr., M. K. Sain, and J. D. Carlson, "An experimental study of MR dampers for seismic protection," Smart Materials and Structures, vol. 7, no. 5, pp. 693-703, 1998.

[2] H. P. Gavin, "Control of seismically excited vibration using electrorheological materials and Lyapunov methods," IEEE Transactions on Control Systems Technology, vol. 9, no. 1, pp. 2736, 2001.

[3] H. Sodeyama, K. Suzuki, and K. Sunakoda, "Development of large capacity semi-active seismic damper using magnetorheological fluid," Journal of Pressure Vessel Technology, Transactions of the ASME, vol. 126, no. 1, pp. 105-109, 2004.

[4] N. Varadarajan and S. Nagarajaiah, "Wind response control of building with variable stiffness tuned mass damper using empirical mode decomposition/Hilbert transform," Journal of Engineering Mechanics, vol. 130, no. 4, pp. 451-458, 2004.

[5] K. Hiramoto, T. Matsuoka, and K. Sunakoda, "Semi-active vibration control of structural systems based on a reference active control law: Output emulation approach," Structural Control and Health Monitoring, vol. 23, no. 3, pp. 423-445, 2016.

[6] M. T. Hagen, H. B. Demuth, and M. H. Beale, Neural Network Design, Ed. PWS Publishing, Boston, MA, USA.

[7] K. Kanai, "Semi-empirical formula for the seismic characteristics of the ground," Univ. Tokyo Bull. Earthquake Res. Inst, vol. 35, pp. 309-325, 1957.

[8] H. Tajimi, "A statistical method of determining the maximum response of a building structure during an earthquake," in proceedings of Proc. 2nd World Conf. Earthquake Eng., pp. 781$798,1960$.

[9] A. Packard and J. Doyle, "The complex structured singular value," Automatica, vol. 29, no. 1, pp. 71-109, 1993.

[10] M. Chilali and P. Gahinet, " $H_{\infty}$ design with pole placement constraints: An LMI approach," IEEE Transactions on Automatic Control, vol. 41, no. 3, pp. 358-367, 1996.

[11] D. E. Rumelhart, G. E. Hinton, and R. J. Williams, "Learning representations by back-propagating errors," Nature, vol. 323, no. 6088, pp. 533-536, 1986.

[12] J. M. Kelly, G. Leitmann, and A. G. Soldatos, "Robust control of base-isolated structures under earthquake excitation," Journal of Optimization Theory and Applications, vol. 53, no. 2, pp. 159180, 1987.

[13] H. Li and J. Ou, "A design approach for semi-active and smart base-isolated buildings," Structural Control and Health Monitoring, vol. 13, no. 2-3, pp. 660-681, 2006. 


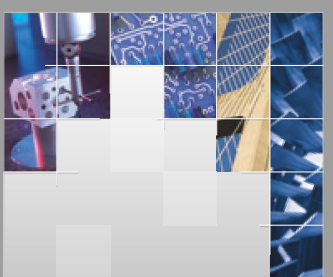

\section{Enfincering}
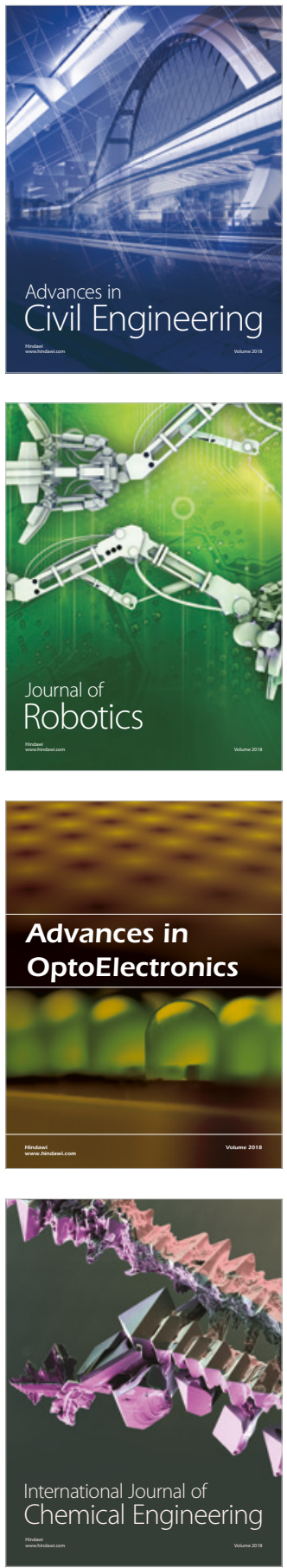

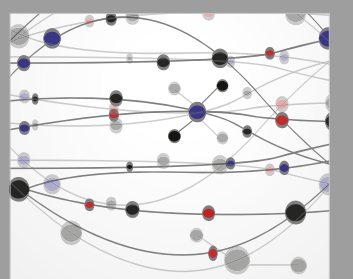

\section{Rotating \\ Machinery}

The Scientific World Journal

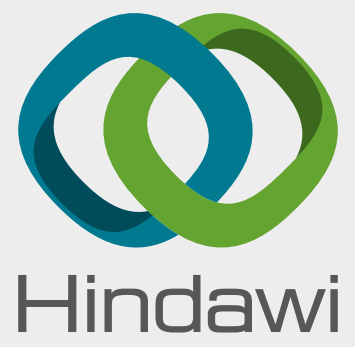

Submit your manuscripts at

www.hindawi.com
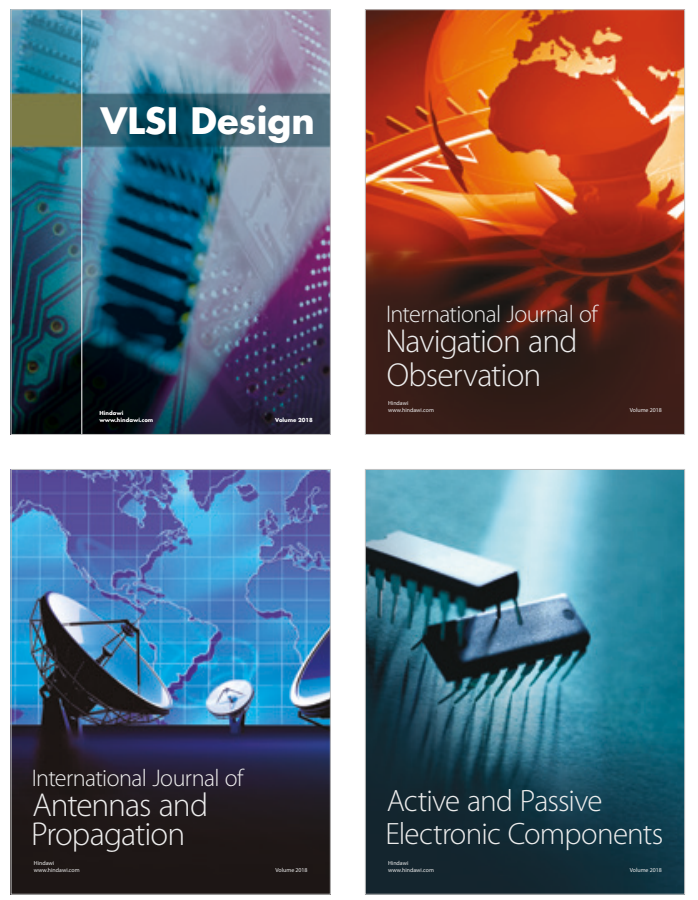
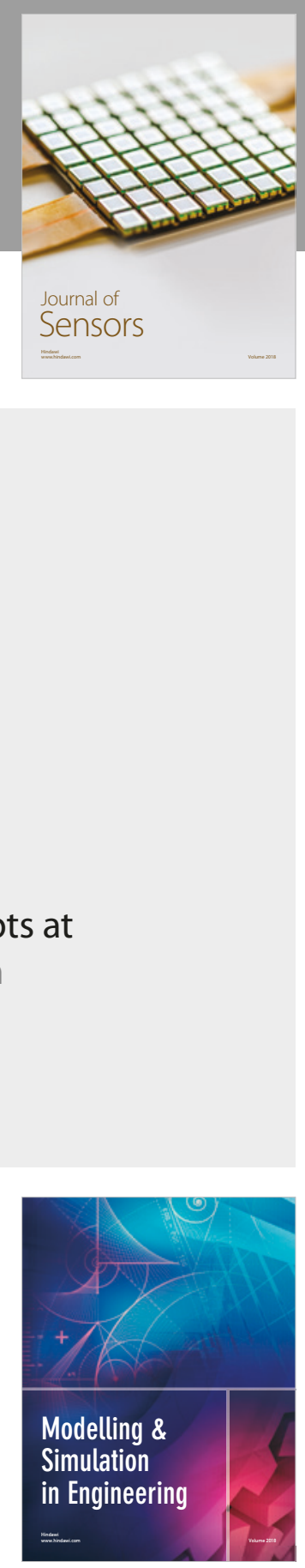

\section{Advances \\ Multimedia}
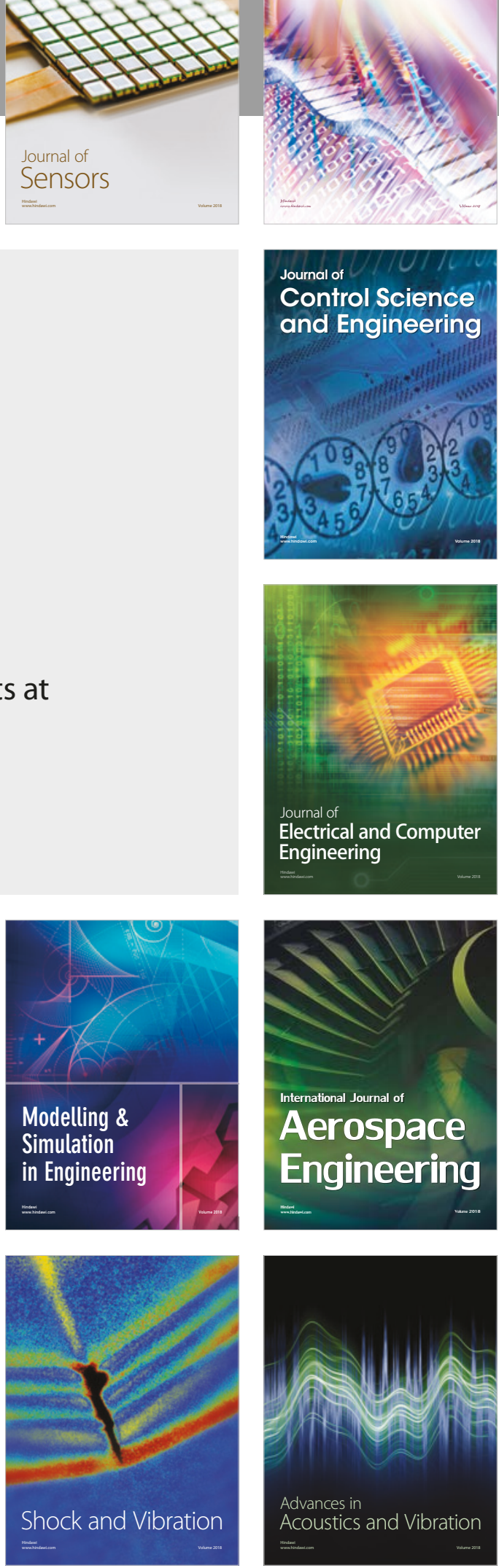